University of Nebraska - Lincoln

DigitalCommons@University of Nebraska - Lincoln

$1-2013$

\title{
Analytic description of elastic electron-atom scattering in an elliptically polarized laser field
}

\author{
A. V. Flegel \\ University of Nebraska-Lincoln, aflegel6@unl.edu
}

M. V. Frolov

Voronezh State University, Russia, frolov@phys.vsu.ru

N. L. Manakov

Voronezh State University, manakov@phys.vsu.ru

Anthony F. Starace

University of Nebraska-Lincoln, astarace1@unl.edu

A. N. Zheltukhin

Voronezh State University, Voronezh, Russia

Follow this and additional works at: https://digitalcommons.unl.edu/physicsstarace

Part of the Atomic, Molecular and Optical Physics Commons, Elementary Particles and Fields and String Theory Commons, and the Plasma and Beam Physics Commons

Flegel, A. V.; Frolov, M. V.; Manakov, N. L.; Starace, Anthony F.; and Zheltukhin, A. N., "Analytic description of elastic electron-atom scattering in an elliptically polarized laser field" (2013). Anthony F. Starace Publications. 193.

https://digitalcommons.unl.edu/physicsstarace/193

This Article is brought to you for free and open access by the Research Papers in Physics and Astronomy at DigitalCommons@University of Nebraska - Lincoln. It has been accepted for inclusion in Anthony F. Starace Publications by an authorized administrator of DigitalCommons@University of Nebraska - Lincoln. 


\title{
Analytic description of elastic electron-atom scattering in an elliptically polarized laser field
}

\author{
A. V. Flegel,,${ }^{1,2}$ M. V. Frolov, ${ }^{3}$ N. L. Manakov, ${ }^{3}$ Anthony F. Starace, ${ }^{1}$ and A. N. Zheltukhin ${ }^{3}$ \\ ${ }^{1}$ Department of Physics and Astronomy, The University of Nebraska, Lincoln, Nebraska 68588-0299, USA \\ ${ }^{2}$ Department of Computer Science, Voronezh State University, Voronezh 394006, Russia \\ ${ }^{3}$ Department of Physics, Voronezh State University, Voronezh 394006, Russia
}

(Received 25 October 2012; published 3 January 2013)

\begin{abstract}
An analytic description of laser-assisted electron-atom scattering (LAES) in an elliptically polarized field is presented using time-dependent effective range (TDER) theory to treat both electron-laser and electron-atom interactions nonperturbatively. Closed-form formulas describing plateau features in LAES spectra are derived quantum mechanically in the low-frequency limit. These formulas provide an analytic explanation for key features of the LAES differential cross section. For the low-energy region of the LAES spectrum, our result generalizes the Kroll-Watson formula to the case of elliptic polarization. For the high-energy (rescattering) plateau in the LAES spectrum, our result generalizes prior results for a linearly polarized field valid for the high-energy end of the rescattering plateau [Flegel et al., J. Phys. B 42, 241002 (2009)] and confirms the factorization of the LAES cross section into three factors: two field-free elastic electron-atom scattering cross sections (with laser-modified momenta) and a laser field-dependent factor (insensitive to the scattering potential) describing the laser-driven motion of the electron in the elliptically polarized field. We present also approximate analytic expressions for the exact TDER LAES amplitude that are valid over the entire rescattering plateau and reduce to the three-factor form in the plateau cutoff region. The theory is illustrated for the cases of $e$ - $\mathrm{H}$ scattering in a $\mathrm{CO}_{2}$-laser field and $e$-F scattering in a midinfrared laser field of wavelength $\lambda=3.5 \mu \mathrm{m}$, for which the analytic results are shown to be in good agreement with exact numerical TDER results.
\end{abstract}

DOI: 10.1103/PhysRevA.87.013404

PACS number(s): $34.80 . \mathrm{Qb}, 34.50 . \mathrm{Rk}, 03.65 . \mathrm{Nk}$

\section{INTRODUCTION}

The interaction of an intense laser field with atoms or molecules results in highly nonlinear processes whose spectra are characterized by plateaulike structures, that is, by a nearly constant dependence of the cross sections on the number $n$ of absorbed photons over a wide interval of $n$. These plateaus are well known for spectra of above-threshold ionization (ATI) and high-order harmonic generation (HHG) [1-3]. The rescattering picture [4-6] provides a transparent physical explanation for the appearance of plateau structures: An intense oscillating laser field returns ionized electrons back to the parent ion, whereupon they either gain additional energy from the laser field during laser-assisted collisional events, thereby forming the high-energy plateau in ATI spectra, or recombine with the parent ion, emitting high-order harmonic photons. High-energy plateaus originating from laser-driven electron rescattering were predicted also for laser-assisted radiative electron-ion recombination or attachment $[7,8]$ and laser-assisted electron-atom scattering (LAES) [9,10]. For laser-induced bound-bound (as in $\mathrm{HHG}$ ) and bound-free (ATI) transitions, rescattering effects are suppressed for an elliptically polarized laser field and completely disappear for circular polarization. In contrast, for laser-assisted collisional processes (such as LAES) a rescattering plateau exists even for a circularly polarized laser field [11] (cf. also Ref. [12]). The classical rescattering scenario used to explain plateaus in LAES spectra for a linearly polarized field has been justified by a quantum-mechanically derived analytic formula for the LAES differential cross section [13], which provides the rescattering correction to the well-known Bunkin-Fedorov [14] and Kroll-Watson [15] results. This formula factorizes the LAES cross section into the product of two field-free cross sections for elastic electron-atom scattering with laser-modified momenta and a "propagation" factor (insensitive to atomic parameters) describing the laser-driven motion of the electron along a closed classical trajectory. These three factors provide closed-form quantum expressions for each of the three steps of the classical rescattering scenario for the LAES process.

Besides its fundamental interest for understanding better the physics of nonlinear phenomena, factorization of the outcomes for nonlinear laser-atom processes in terms of laser-dependent factors and factors describing the field-free atomic dynamics provides an efficient means for retrieving these atomic factors from measured spectra of strong-field processes. At present, such factorizations form the basis for HHG and ATI spectroscopies that allow the retrieval of the photoionization cross sections for the outer electron shells of atoms or molecules (from HHG spectra) (cf., e.g., Ref. [16]) and differential cross sections of elastic electron scattering from the positive ion of a target (from ATI spectra) (cf., e.g., Refs. [17,18]). The factorization of HHG and ATI yields was first postulated based on numerical solutions of the time-dependent Schrödinger equation [19] (cf. also the review [20]) and was then justified theoretically [within the time-dependent effective range (TDER) theory [21,22] for the case of a monochromatic field in Refs. [23,24] for HHG and in Ref. [25] for ATI, and for the case of a short laser pulse in Refs. [26] (for HHG) and [27] (for ATI). We note that in all the aforementioned studies only linearly polarized laser fields were considered, in which case the theoretical treatment is simplified (due to the one-dimensional laser-driven propagation of the active electron along the direction of laser polarization). However, although the driving laser ellipticity provides an additional control parameter for intense laser-atom interactions, at present there does not exist a convincing justification for the factorization of the rates 
or cross sections of nonlinear phenomena in an elliptically polarized field, neither for laser-induced nor for laser-assisted processes.

In this paper we show analytically that the LAES cross section in the region of the rescattering plateau cutoff may be expressed in factorized form (as the product of three factors) for the general case of an elliptically polarized laser field. This result generalizes that for the case of linear polarization [13] and presents a rare example of a strong-field process whose yield may be factorized for the case of a nonzero driving laser ellipticity. The results presented are obtained taking into account the rescattering effects nonperturbatively within the TDER theory for collision problems [28] as reformulated for the case of LAES in a low-frequency, elliptically polarized field. Based on a detailed analysis of the two-dimensional closed classical trajectories of an electron in the laser polarization plane, we have obtained also an analytic estimate for the (nonfactorized) LAES amplitude that describes the entire energy region of the rescattering plateau. Our analytic results are in good agreement with exact numerical TDER results.

The paper is organized as follows. In Sec. II we provide the basic results of the TDER theory for the scattering state of an electron as well as for the LAES amplitude in an elliptically polarized laser field. In Sec. III we develop a low-frequency expansion for the key ingredient of TDER theory: the periodic function of time, $f_{\mathbf{p}}(t)$, that enters the TDER result for the scattering state. This expansion allows one to approximate the scattering state as a sum of two terms: a zero-order ("Kroll-Watson") term and a rescattering correction, which is responsible for the high-energy plateau in the LAES spectrum. The low-energy part of the LAES spectrum, described by the Kroll-Watson term in the LAES amplitude, is considered in Sec. IV, while in Sec. V we provide a detailed analysis of the LAES amplitude in the rescattering approximation, that is, including the rescattering correction. In Sec. VI we present the factorized (three-factor) form for the LAES cross section in the rescattering approximation, compare the LAES spectra in this approximation with exact TDER results, and discuss the influence of the laser ellipticity on key features of LAES spectra. Some conclusions and perspectives for further use of the TDER theory for description of LAES in an elliptically polarized field are discussed briefly in Sec. VII. Finally, in two appendixes we present an alternative representation for the TDER LAES amplitude that we use for the exact numerical calculations within the TDER theory (Appendix A) and a brief description of the uniform asymptotic approximation of an integral involving a highly oscillatory function (Appendix B).

\section{BASIC EQUATIONS OF THE TDER THEORY FOR LAES}

\section{A. Formulation of the problem}

We consider the scattering of an incoming electron having momentum $\mathbf{p}$ and kinetic energy $E=p^{2} /(2 m)$ on a target atom in the presence of a long laser pulse approximated by a monochromatic, elliptically polarized plane wave having intensity $I$ and frequency $\omega$. We assume that both the electron energy $E$ and the laser photon energy $\hbar \omega$ are small compared to atomic excitation energies and that the laser parameters $I$ and $\omega$ are such that laser excitation or ionization of atomic electrons is negligible. Under these assumptions, the electron-atom interaction can be approximated by a short-range potential $U(r)$ (that vanishes for $r \gtrsim r_{c}$ ). Thus, the LAES process can be described as potential (elastic) electron scattering accompanied by absorption or emission of $n$ laser photons (with $n_{\min }=-[E /(\hbar \omega)]$, where $[x]$ is the integer part of $x$ ). Thus, the momentum (or energy) spectra of the scattered electrons (the LAES spectra) are characterized by momenta $\mathbf{p}_{n}$ and energies $E_{n}=p_{n}^{2} /(2 m)=E+n \hbar \omega$.

For the electron-laser interaction, we use the dipole approximation in the length gauge,

$$
V(\mathbf{r}, t)=-e \mathbf{r} \cdot \mathbf{F}(t),
$$

where $\mathbf{F}(t)$ is the electric vector of the laser field,

$$
\mathbf{F}(t)=F \operatorname{Re}\left(\mathbf{e} e^{-i \omega t}\right), \quad \mathbf{e} \cdot \mathbf{e}^{*}=1 .
$$

The complex polarization unit vector $\mathbf{e}$ in Eq. (2) is parametrized as

$$
\mathbf{e}=\frac{\hat{\boldsymbol{\epsilon}}+i \eta[\hat{\boldsymbol{\kappa}} \times \hat{\boldsymbol{\epsilon}}]}{\sqrt{1+\eta^{2}}}, \quad-1 \leqslant \eta \leqslant 1,
$$

where $\hat{\boldsymbol{\epsilon}}$ is a unit vector along the major axis of the polarization ellipse, the unit vector $\hat{\kappa}$ defines the laser propagation direction, and $\eta$ is the ellipticity. With the definition (3), the laser intensity does not depend on $\eta: I=c F^{2} /(8 \pi)$. Along with $\eta$, the degrees of linear $(\ell)$ and circular $(\xi)$ polarization are convenient parameters for describing an elliptically polarized field:

$$
\ell=\mathbf{e} \cdot \mathbf{e}=\frac{1-\eta^{2}}{1+\eta^{2}}, \quad \xi=i \hat{\kappa} \cdot\left[\mathbf{e} \times \mathbf{e}^{*}\right]=\frac{2 \eta}{1+\eta^{2}} .
$$

Note that the scalar product of the polarization vector $\mathbf{e}$ with a unit vector $\mathbf{u}$, defined by the two spherical angles, $\theta_{\mathbf{u}}$ and $\phi_{\mathbf{u}}$, as $\mathbf{u}=\left(\hat{\boldsymbol{\epsilon}} \cos \phi_{\mathbf{u}}+[\hat{\boldsymbol{\kappa}} \times \hat{\boldsymbol{\epsilon}}] \sin \phi_{\mathbf{u}}\right) \sin \theta_{\mathbf{u}}+\hat{\boldsymbol{\kappa}} \cos \theta_{\mathbf{u}}$, is complex and can be parametrized as

$$
\begin{aligned}
\mathbf{u} \cdot \mathbf{e} & =|\mathbf{u} \cdot \mathbf{e}| e^{i \varphi_{\mathbf{u}}}, \quad \varphi_{\mathbf{u}} \equiv \arg (\mathbf{u} \cdot \mathbf{e}), \\
|\mathbf{u} \cdot \mathbf{e}| & =\sin \theta_{\mathbf{u}} \sqrt{\left(1+\ell \cos 2 \phi_{\mathbf{u}}\right) / 2}, \\
\tan \varphi_{\mathbf{u}} & =\eta \tan \phi_{\mathbf{u}} .
\end{aligned}
$$

For an analytic nonperturbative account of both the electron-laser and the electron-atom interactions in electron scattering assisted by a low-frequency elliptically polarized laser field, we adapt the TDER theory [28] for LAES to the case of a low-frequency field. The atomic potential $U(r)$ is assumed to support a single (negative ion) weakly bound state $\psi_{\kappa l m_{l}}(\mathbf{r})$ with energy $E_{0}=-\hbar^{2} \kappa^{2} /(2 m)\left(\kappa r_{c} \ll 1\right)$ and angular momentum $l$. In particular, $l=0$ corresponds to electron scattering from hydrogen or an alkali-metal atom, and $l=1$ corresponds to a halogen atom target.

The key idea of the TDER theory is the same as in effective range theory for two stationary potentials, $U(r)$ and $V(\mathbf{r})$, which exert their influence on the electron predominantly in two essentially nonoverlapping coordinate ranges [29]: $U(r)$ is important primarily for $r \lesssim r_{c}$, while a long-range, externalfield potential $V(\mathbf{r})$ is important primarily for $r \gg \kappa^{-1}$. Thus, in the region $r_{c} \lesssim r \ll \kappa^{-1}$, the low-energy electron may be considered as virtually free. In this case, as in effective range theory for low-energy electron scattering [30], only a single parameter, the $l$-wave scattering phase $\delta_{l}$ for the potential $U(r)$, 
determines the $l$-wave component of the exact scattering state $\psi_{\mathbf{p}}(\mathbf{r})$ in the region $r_{c} \lesssim r \ll \min \left(\kappa^{-1}, k^{-1}\right)(k=\sqrt{2 m E} /$ $\hbar=p / \hbar)$ :

$$
\int Y_{l m_{l}}^{*}(\hat{\mathbf{r}}) \psi_{\mathbf{p}}(\mathbf{r}) d \Omega_{\mathbf{r}} \sim r^{-l-1}+\cdots+B_{l}(E)\left(r^{l}+\cdots\right),
$$

where the factor $B_{l}(E)$ involves the phase shift $\delta_{l}(k)$ and can be approximated by the two fundamental parameters of effective range theory: the scattering length $\left(a_{l}\right)$ and the effective range $\left(r_{l}\right)$ :

$$
\begin{aligned}
(2 l-1) ! !(2 l+1) ! ! B_{l}(E) & \equiv k^{2 l+1} \cot \delta_{l}(k) \\
& =-a_{l}^{-1}+r_{l} k^{2} / 2 .
\end{aligned}
$$

The boundary condition (6) for $\psi_{\mathbf{p}}(\mathbf{r})$ at small $r$ is the key equation that allows one to represent the scattering state $\psi_{\mathbf{p}}(\mathbf{r})$ outside the potential $U(r)$ (i.e., for $r \gtrsim r_{c}$ ) in terms of the two parameters of the problem, $a_{l}$ and $r_{l}$, independent of the shape of $U(r)$.

\section{B. Scattering state of an electron in TDER theory}

We seek the laser-dressed scattering state, $\Psi_{\mathbf{p}}(\mathbf{r}, t)$, of an electron in the LAES process using the Floquet or quasienergy state (QES) representation (cf., e.g., Ref. [31]):

$$
\Psi_{\mathbf{p}}(\mathbf{r}, t)=e^{-i \epsilon t / \hbar} \Phi_{\mathbf{p}}(\mathbf{r}, t), \quad \Phi_{\mathbf{p}}(\mathbf{r}, t)=\Phi_{\mathbf{p}}(\mathbf{r}, t+2 \pi / \omega),
$$

where $\epsilon=E+u_{p}$ is the quasienergy and $u_{p}=e^{2} F^{2} /\left(4 m \omega^{2}\right)$ is the ponderomotive (or quiver) energy. The QES wave function $\Phi_{\mathbf{p}}(\mathbf{r}, t)$ is a periodic solution of the time-dependent Schrödinger equation:

$$
\left(i \hbar \frac{\partial}{\partial t}+\epsilon+\frac{\hbar^{2}}{2 m} \Delta-U(r)-V(\mathbf{r}, t)\right) \Phi_{\mathbf{p}}(\mathbf{r}, t)=0 .
$$

Owing to the time dependence of $\Phi_{\mathbf{p}}(\mathbf{r}, t)$, the boundary condition for the $l$-wave component of $\Phi_{\mathbf{p}}(\mathbf{r}, t)$ at small $r \gtrsim r_{c}$ must be modified compared to Eq. (6) by introducing some time-periodic functions (as was done similarly in TDER theory for bound states in an elliptically polarized field [21,22]). Since $V(\mathbf{r}, t)$ lacks axial symmetry in the case of an elliptically polarized field, the $l$-wave component of $\Phi_{\mathbf{p}}(\mathbf{r}, t)$ depends, in general, on the angular momentum projection $m_{l}$. However, for small $r \gtrsim r_{c}$ the potentials $U(r)$ and $V(\mathbf{r}, t)$ can be neglected in Eq. (9), so that the $l$-wave component of any time-periodic solution of Eq. (9) is independent of $m_{l}$ and may be written as

$$
\int Y_{l m_{l}}^{*}(\hat{\mathbf{r}}) \Phi_{\mathbf{p}}(\mathbf{r}, t) d \Omega_{\mathbf{r}}=\sum_{k}\left[a_{k} j_{l}\left(\varkappa_{k} r\right)+b_{k} y_{l}\left(\varkappa_{k} r\right)\right] e^{-i k \omega t},
$$

where $\varkappa_{k}=\sqrt{2 m(\epsilon+k \hbar \omega)} / \hbar, j_{l}$ and $y_{l}$ are the regular and irregular spherical Bessel functions (behaving respectively as $\sim r^{l}$ and $\sim r^{-l-1}$ as $\left.r \rightarrow 0\right)$, and $a_{k}$ and $b_{k}$ are constants. Replacing $j_{l}\left(\varkappa_{k} r\right)$ and $y_{l}\left(\varkappa_{k} r\right)$ in Eq. (10) by their expansions for $\varkappa_{k} r \ll 1$, defining the factor $B_{l}(\epsilon+k \hbar \omega)$ as proportional to the coefficient ratio $a_{k} / b_{k}$, and introducing coefficients $f_{k}^{\left(l m_{l}\right)}$, in which the index $m_{l}$ labels the angular momentum projection onto $Y_{l m_{l}}$ on the left of Eq. (10), we obtain a generalization of the boundary condition (6) for a time-dependent interaction
$V(\mathbf{r}, t):$

$$
\begin{aligned}
& \int Y_{l m_{l}}^{*}(\hat{\mathbf{r}}) \Phi_{\mathbf{p}}(\mathbf{r}, t) d \Omega_{\mathbf{r}} \\
& \sim \sum_{k}\left[r^{-l-1}+\cdots+B_{l}(\epsilon+k \hbar \omega)\left(r^{l}+\cdots\right)\right] f_{k}^{\left(l m_{l}\right)} e^{-i k \omega t} \\
& =\left[r^{-l-1}+\cdots+B_{l}(\epsilon)\left(r^{l}+\cdots\right)\right] f_{\mathbf{p}}^{\left(l m_{l}\right)}(t) \\
& \quad+i\left(r^{l}+\cdots\right) \frac{(2 l+1)}{[(2 l+1) ! !]^{2}} \frac{r_{l} m}{\hbar} \frac{d}{d t} f_{\mathbf{p}}^{\left(l m_{l}\right)}(t)
\end{aligned}
$$

where the effective range parametrization (7) for $B_{l}(\epsilon+k \hbar \omega)$ was substituted on the left of the equality in Eq. (11) in order to obtain the final result summed over $k$ on the right in terms of the time-periodic function:

$$
f_{\mathbf{p}}^{\left(l m_{l}\right)}(t)=\sum_{k} f_{k}^{\left(l m_{l}\right)} e^{-i k \omega t} .
$$

The desired solution of the exact equation (9) for the scattering states has the general form

$$
\Phi_{\mathbf{p}}(\mathbf{r}, t)=\chi_{\mathbf{p}}(\mathbf{r}, t)+\Phi_{\mathbf{p}}^{(\mathrm{sc})}(\mathbf{r}, t),
$$

where the "scattered wave" $\Phi_{\mathbf{p}}^{(\mathrm{sc})}(\mathbf{r}, t)$ is an outgoing wave at $r \rightarrow \infty$, while the "incident wave" $\chi_{\mathbf{p}}(\mathbf{r}, t)$ is the QES wave function of a free electron with momentum $\mathbf{p}$ in the laser field (i.e., the time-periodic part of a Volkov wave function),

$$
\chi_{\mathbf{p}}(\mathbf{r}, t)=e^{i\left[\mathbf{r} \cdot \mathbf{P}(t)-S_{\mathbf{p}}(t)\right] / \hbar},
$$

where

$$
\begin{aligned}
S_{\mathbf{p}}(t) & =\int^{t}\left[\mathbf{P}^{2}(\tau) /(2 m)-\epsilon\right] d \tau \\
& =-\mathbf{p} \cdot \frac{e \mathbf{F}(t)}{m \omega^{2}}+\int^{t}\left[\frac{e^{2} \mathbf{A}^{2}(\tau)}{2 m c^{2}}-u_{p}\right] d \tau,
\end{aligned}
$$

and $\mathbf{P}(t)=\mathbf{p}-(e / c) \mathbf{A}(t)$ is the electron's kinetic momentum in the laser field $\mathbf{F}(t)$ with vector potential $\mathbf{A}(t)$, where $\mathbf{F}(t)=-c^{-1} d \mathbf{A}(t) / d t$.

According to the TDER theory [28], the function $\Phi_{\mathbf{p}}^{(\mathrm{sc})}(\mathbf{r}, t)$ in the outer region, $r \gtrsim r_{c}$ [in which the potential $U(r)$ vanishes], can be expressed in terms of the retarded Green's function $G\left(\mathbf{r}, t ; \mathbf{r}, t^{\prime}\right)$ of a free electron in the laser field $\mathbf{F}(t)$ and involves the function $f_{\mathbf{p}}^{\left(l m_{l}\right)}(t)$ in the boundary condition (11). [Indeed, upon neglecting $U(r)$, any solution of Eq. (9) can be represented as a wave packet composed of wave functions for a free electron in the field $\mathbf{F}(t)$.] For $G\left(\mathbf{r}, t ; \mathbf{r}, t^{\prime}\right)$ we use the well-known Feynman form,

$$
\begin{aligned}
G\left(\mathbf{r}, t ; \mathbf{r}^{\prime}, t^{\prime}\right)= & -\theta\left(t-t^{\prime}\right) \frac{i}{\hbar}\left[\frac{m}{2 \pi i \hbar\left(t-t^{\prime}\right)}\right]^{3 / 2} \\
& \times \exp \left[i S\left(\mathbf{r}, t ; \mathbf{r}^{\prime}, t^{\prime}\right) / \hbar\right],
\end{aligned}
$$

where $\theta(x)$ is Heaviside function and $S$ is the classical action for an electron in the laser field $\mathbf{F}(t)$ :

$$
\begin{aligned}
S\left(\mathbf{r}, t ; \mathbf{r}^{\prime}, t^{\prime}\right)= & \frac{m}{2\left(t-t^{\prime}\right)}\left(\mathbf{r}-\mathbf{r}^{\prime}+\frac{e}{m \omega^{2}}\left[\mathbf{F}(t)-\mathbf{F}\left(t^{\prime}\right)\right]\right)^{2} \\
& -\frac{e^{2}}{2 m c^{2}} \int_{t^{\prime}}^{t} \mathbf{A}^{2}(\tau) d \tau-\frac{e}{c}\left[\mathbf{r} \cdot \mathbf{A}(t)-\mathbf{r}^{\prime} \cdot \mathbf{A}\left(t^{\prime}\right)\right] .
\end{aligned}
$$


The behavior of $\Phi_{\mathbf{p}}(\mathbf{r}, t)$ as $r \rightarrow 0$ required by the condition (11) [namely, the $l$-wave component of $\Phi_{\mathbf{p}}(\mathbf{r}, t)$ should involve a singular term $\left.\sim r^{-l-1} Y_{l m_{l}}(\hat{\mathbf{r}})\right]$ may be ensured by $l$-fold differentiation of $G\left(\mathbf{r}, t ; \mathbf{r}, t^{\prime}\right)$ over $\mathbf{r}^{\prime}$ followed by the substitution $\mathbf{r}^{\prime}=0$. [From the explicit form (16) of $G$, such differentiation does not change the asymptotic behavior of $\Phi_{\mathbf{p}}(\mathbf{r}, t)$ for $r \rightarrow \infty$.] As a result, in a way similar to that for the TDER treatment of a quasistationary QES with an initial angular momentum $l[21,22]$, the general TDER expression for $\Phi_{\mathbf{p}}^{(\mathrm{sc})}(\mathbf{r}, t)$ can be written as follows [28]:

$$
\begin{aligned}
\Phi_{\mathbf{p}}^{(\mathrm{scc})}(\mathbf{r}, t)= & -\frac{2 \pi \hbar^{2}}{m \kappa^{1+l}} \sum_{\mu=-l}^{l} \int_{-\infty}^{t} d t^{\prime} e^{i \epsilon\left(t-t^{\prime}\right) / \hbar} f_{\mathbf{p}}^{(l \mu)}\left(t^{\prime}\right) \\
& \times\left.\mathcal{Y}_{l \mu}\left(\nabla_{\mathbf{r}^{\prime}}\right) G\left(\mathbf{r}, t ; \mathbf{r}^{\prime}, t^{\prime}\right)\right|_{\mathbf{r}^{\prime}=0},
\end{aligned}
$$

where the differential operator $\mathcal{Y}_{l \mu}\left(\nabla_{\mathbf{r}}\right)$ is obtained from the solid harmonic $\mathcal{Y}_{l \mu}(\mathbf{r})\left[\equiv r^{l} Y_{l \mu}(\hat{\mathbf{r}})\right]$ by the substitution $\mathbf{r} \rightarrow \nabla_{\mathbf{r}}$. Equations for the unknown functions $f_{\mathbf{p}}^{(l \mu)}(t)$ complete the construction of the scattering state $\Phi_{\mathbf{p}}(\mathbf{r}, t)$ in TDER theory [cf. Eqs. (13) and (18)]. These equations are obtained by matching the $l$-wave components of $\Phi_{\mathbf{p}}(\mathbf{r}, t)$ [which are different for different values of $m_{l}$, as noted above and as is clear from the explicit representation (18) for $\left.\Phi_{\mathbf{p}}^{(\mathrm{sc})}(\mathbf{r}, t)\right]$ at small $r$ to the prescribed boundary condition (11). Due to the term $\chi_{\mathbf{p}}(\mathbf{r}, t)$ in Eq. (13), the resulting equations comprise a system of $2 l+1$ coupled inhomogeneous integro-differential equations for the functions $f_{\mathbf{p}}^{\left(l m_{l}\right)}(t)$, with $m_{l}=-l, \ldots, l$. Because the derivation and analysis of these equations involve the same steps for both $l>0$ and $l=0$ (differing only in the complexity of the analytical transformations), for greater clarity, in the rest of this paper we provide analytical derivations only for the case of $l=0$ (" $s$-wave scattering"). (For an analytical treatment of a similar, though homogeneous, system of equations in TDER theory for bound states with $l>0$, see Refs. [21,22].)

\section{Exact TDER LAES amplitude and differential cross section for $s$-wave scattering}

If the potential $U(r)$ supports only a single weakly bound $s$ state so that only the phase shift $\delta_{0}(k)$ is nonzero, then Eqs. (11) and (18) simplify as follows:

$$
\begin{aligned}
\Phi_{\mathbf{p}}^{(\mathrm{sc})}(\mathbf{r}, t) & =-\frac{2 \pi \hbar^{2}}{m \kappa} \int_{-\infty}^{t} d t^{\prime} e^{i \epsilon\left(t-t^{\prime}\right) / \hbar} f_{\mathbf{p}}\left(t^{\prime}\right) G\left(\mathbf{r}, t ; 0, t^{\prime}\right), \\
\Phi_{\mathbf{p}}(\mathbf{r}, t) & \sim\left(\frac{1}{r}+B_{0}(\epsilon)\right) f_{\mathbf{p}}(t)+i \frac{r_{0} m}{\hbar} \frac{d}{d t} f_{\mathbf{p}}(t),
\end{aligned}
$$

where $f_{\mathbf{p}}(t) \equiv f_{\mathbf{p}}^{(00)}(t)$ and

$$
B_{0}(\epsilon)=-a_{0}^{-1}+r_{0} m \epsilon / \hbar^{2} .
$$

To match the function $\Phi_{\mathbf{p}}(\mathbf{r}, t)$ [cf. Eqs. (13) and (19)] to the $r \rightarrow 0$ boundary condition (20), we extract from the integrand in Eq. (19) a term proportional to the field-free Green's function $G_{0}\left(\mathbf{r}, t ; 0, t^{\prime}\right)$ [given by Eq. (16) with $\mathbf{F}(t)=0$ ]:

$$
\begin{aligned}
\Phi_{\mathbf{p}}^{(\mathrm{sc})}(\mathbf{r}, t)= & -\frac{2 \pi \hbar^{2}}{m \kappa} \int_{-\infty}^{t} d t^{\prime}\left[e^{i \epsilon\left(t-t^{\prime}\right) / \hbar} f_{\mathbf{p}}\left(t^{\prime}\right) G\left(\mathbf{r}, t ; 0, t^{\prime}\right)\right. \\
& \left.-f_{\mathbf{p}}(t) G_{0}\left(\mathbf{r}, t ; 0, t^{\prime}\right)\right]+\frac{1}{\kappa r} f_{\mathbf{p}}(t) .
\end{aligned}
$$

The integral in Eq. (22) is now regular at $\mathbf{r}=0$. Setting then $\mathbf{r}=0$ in $\chi_{\mathbf{p}}(\mathbf{r}, t)$, comparing the result for $\Phi_{\mathbf{p}}(\mathbf{r}, t)$ at small $r$ with Eq. (20), and introducing the dimensionless time $\tau=\omega t$, we obtain an inhomogeneous integro-differential equation for $f_{\mathbf{p}}(\tau) \equiv f_{\mathbf{p}}(t=\tau / \omega)$ :

$$
\begin{aligned}
B_{0}(\epsilon) f_{\mathbf{p}}(\tau)+i & \frac{r_{0} m \omega}{\hbar} \frac{d}{d \tau} f_{\mathbf{p}}(\tau)=\kappa e^{-i S_{\mathbf{p}}(\tau) / \hbar}+\mathcal{I}\left[f_{\mathbf{p}}(\tau)\right], \\
\mathcal{I}\left[f_{\mathbf{p}}(\tau)\right]= & \sqrt{\frac{m \omega}{2 \pi i \hbar}} \int_{0}^{\infty} \frac{d x}{x^{3 / 2}}\left[e^{(i / \hbar)[\epsilon x / \omega+S(\tau, \tau-x)]}\right. \\
& \left.\times f_{\mathbf{p}}(\tau-x)-f_{\mathbf{p}}(\tau)\right],
\end{aligned}
$$

where $S_{\mathbf{p}}(\tau) \equiv S_{\mathbf{p}}(t=\tau / \omega)$, and $S\left(\tau, \tau^{\prime}\right) \equiv S(\mathbf{r}=0, t=\tau / \omega$; $\left.\mathbf{r}^{\prime}=0, t^{\prime}=\tau^{\prime} / \omega\right)$.

As is usual, the LAES amplitude $\mathcal{A}_{n}\left(\mathbf{p}, \mathbf{p}_{n}\right)$ is determined by the asymptotic behavior of the wave function $\Phi_{\mathbf{p}}^{(\mathrm{sc})}(\mathbf{r}, t)$ in Eq. (18) as $r \rightarrow \infty$. For $s$-wave scattering, this behavior has the form

$$
\left.\Phi_{\mathbf{p}}^{(\mathrm{sc})}(\mathbf{r}, t)\right|_{\kappa r \gg 1} \simeq e^{-i \phi(\mathbf{r}, t) / \hbar} \sum_{n \geqslant n_{\min }} \mathcal{A}_{n}\left(\mathbf{p}, \mathbf{p}_{n}\right) \frac{e^{i p_{n}|\mathbf{R}(\mathbf{r}, t)| / \hbar-i n \omega t}}{|\mathbf{R}(\mathbf{r}, t)|},
$$

where

$$
\begin{aligned}
\phi(\mathbf{r}, t) & =\frac{e}{c} \mathbf{r} \cdot \mathbf{A}(t)+\int^{t}\left(\frac{e^{2} A^{2}(\tau)}{2 m c^{2}}-u_{p}\right) d \tau, \\
\mathbf{R}(\mathbf{r}, t) & =\mathbf{r}+\frac{e}{m \omega^{2}} \mathbf{F}(t),
\end{aligned}
$$

and the summation over $n$ involves all open channels with exchange of $n$ photons, for which $E_{n}=E+n \hbar \omega>0$. The LAES amplitude $\mathcal{A}_{n}\left(\mathbf{p}, \mathbf{p}_{n}\right)$ may be expressed in terms of $f_{\mathbf{p}}(\tau)$,

$$
\mathcal{A}_{n}\left(\mathbf{p}, \mathbf{p}_{n}\right)=\frac{1}{2 \pi \kappa} \int_{0}^{2 \pi} e^{i n \tau+i S_{\mathbf{p}_{n}}(\tau) / \hbar} f_{\mathbf{p}}(\tau) d \tau,
$$

and the differential LAES cross section is given by

$$
\frac{d \sigma_{n}\left(\mathbf{p}, \mathbf{p}_{n}\right)}{d \Omega_{\mathbf{p}_{n}}}=\frac{p_{n}}{p}\left|\mathcal{A}_{n}\left(\mathbf{p}, \mathbf{p}_{n}\right)\right|^{2} .
$$

For $\mathbf{F}(t)=0$, the function $f_{\mathbf{p}}(\mathbf{F}(t)=0 ; \tau) \equiv f_{0}(p)$ reduces to the amplitude $\mathcal{A}(p)$ for field-free $s$-wave elastic electron scattering on the potential $U(r)$ in the effective range approximation (in which $k=p / \hbar$ ),

$$
f_{0}(p)=\kappa \mathcal{A}(p), \quad \mathcal{A}(p)=\frac{1}{-a_{0}^{-1}+r_{0} k^{2} / 2-i k} .
$$

For $\mathbf{F}(t) \neq 0$, the function $f_{\mathbf{p}}(\tau)$ is a key object of TDER theory, since it contains complete information on the modification of the electron-atom interaction by an elliptically polarized laser field in all LAES channels. Numerical evaluation of $f_{\mathbf{p}}(\tau)$ is done most easily by converting the integro-differential Eq. (23) to a set of inhomogeneous linear algebraic equations for the Fourier coefficients $f_{k}$ of $f_{\mathbf{p}}(\tau)$ [cf. Eq. (A1) in Appendix A]. The LAES amplitude $\mathcal{A}_{n}\left(\mathbf{p}, \mathbf{p}_{n}\right)$ is then expressed in terms of $f_{k}$ and generalized Bessel functions [cf. Eq. (A6)]. As follows from the boundary condition (11) that determines the QES $\Phi_{\mathbf{p}}(\mathbf{r}, t)$ at small $r$ [where the potential $U(r)$ is most important], physically, the coefficients $f_{k}$ govern the population of QES harmonics of the scattering state $\Psi_{\mathbf{p}}(\mathbf{r}, t)$ 
with energies $\epsilon+k \hbar \omega$ that arise as a result of atomic-potentialmediated exchange of $k$ photons between the electron and the laser field at small $r$.

For $s$-wave scattering, the numerical results in this paper, referred to as "exact TDER results," are obtained by numerical solution of Eq. (A1), followed by evaluation of the amplitude $\mathcal{A}_{n}\left(\mathbf{p}, \mathbf{p}_{n}\right)$ according to Eq. (A6). For $p$-wave scattering $(l=1)$, the Fourier coefficients $f_{k}^{(1 m)}$ (where $m=0, \pm 1$ ) of the periodic function (12) satisfy the system of Eqs. (A8) and (A9), while the LAES amplitude is given by Eq. (A13).

An analytic evaluation of the LAES amplitude $\mathcal{A}_{n}\left(\mathbf{p}, \mathbf{p}_{n}\right)$ can be performed in the low-frequency limit, in which case the low-frequency expansion for the solution $f_{\mathbf{p}}(\tau)$ of Eq. (23) can be obtained.

\section{LOW-FREQUENCY EXPANSION OF $\boldsymbol{f}_{\mathrm{p}}(\tau)$}

Because the classical actions $S_{\mathbf{p}}(\tau)$ and $S\left(\tau, \tau^{\prime}\right)$ in the inhomogeneous and integral terms of Eq. (23) oscillate with large amplitudes $\left(\sim u_{p} / \omega\right)$ for the case of an intense lowfrequency field $\mathbf{F}(t)$, we seek the solution $f_{\mathbf{p}}(\tau)$ of Eq. (23) in the following form:

$$
f_{\mathbf{p}}(\tau)=g_{\mathbf{p}}(\tau) e^{-i \int^{\tau} d \tau^{\prime}\left[\mathcal{E}\left(\tau^{\prime}\right)-\epsilon\right] /(\hbar \omega)},
$$

where $g_{\mathbf{p}}(\tau)$ and $\mathcal{E}(\tau)$ are smooth functions satisfying, respectively, the requirements that $\left|d g_{\mathbf{p}} / d \tau\right| \ll u_{p} /(\hbar \omega)$ and that the upper bound of $\mathcal{E}(\tau)$ is of the order of $u_{p}$.

Before proceeding with an iterative solution of Eq. (23), we analyze first the low-frequency limit of the integral term $\mathcal{I}\left[f_{\mathbf{p}}(\tau)\right]$ defined in Eq. (24). For $u_{p} \gg \hbar \omega$, the dominant contribution to the integral (24) comes from the neighborhood of the singular point $x=0$, while the contribution from the domain $x>0$ can be evaluated using the saddle-point method. Thus, we approximate the integral $\mathcal{I}$ [after substituting there Eq. (29)] as a sum:

$$
\mathcal{I}\left[f_{\mathbf{p}}(\tau)\right] \approx \mathcal{I}^{(0)}\left[f_{\mathbf{p}}(\tau)\right]+\sum_{s} \mathcal{I}_{s}\left[f_{\mathbf{p}}(\tau)\right],
$$

where integrals $\mathcal{I}^{(0)}$ and $\mathcal{I}_{s}$ are evaluated, respectively, over the vicinity of $x=0$ and at the saddle points $x=x_{s}>0$. In order to evaluate the term $\mathcal{I}^{(0)}$, we neglect the action $S(\tau, \tau-x)$ (which is of order $x^{3}$ when $x \rightarrow 0$ ) in the integrand of Eq. (24) and approximate the function $f_{\mathbf{p}}(\tau-x)$ for $x \ll 1$ as

$$
f_{\mathbf{p}}(\tau-x) \approx f_{\mathbf{p}}(\tau) e^{i x[\mathcal{E}(\tau)-\epsilon] /(\hbar \omega)} .
$$

We thus obtain for $\mathcal{I}^{(0)}$ the following result:

$$
\begin{aligned}
\mathcal{I}^{(0)}\left[f_{\mathbf{p}}(\tau)\right] & =f_{\mathbf{p}}(\tau) \sqrt{\frac{m \omega}{2 \pi i \hbar}} \int_{0}^{\infty} \frac{d x}{x^{3 / 2}}\left(e^{i x \mathcal{E}(\tau) / \hbar \omega)}-1\right) \\
& =i f_{\mathbf{p}}(\tau) \sqrt{2 m \mathcal{E}(\tau)} / \hbar
\end{aligned}
$$

The result for $\mathcal{I}_{s}$ is obtained by substituting Eq. (29) into Eq. (24), followed by evaluation of $\mathcal{I}$ by the saddle-point method:

$$
\mathcal{I}_{s}\left[f_{\mathbf{p}}(\tau)\right]=f_{\mathbf{p}}\left(\tau-x_{s}\right) \frac{e^{(i / \hbar)\left[\epsilon x_{s} / \omega+S\left(\tau, \tau-x_{s}\right)\right]}}{\alpha_{0}\left[x_{s}^{3} \beta\left(\tau, x_{s}\right)\right]^{1 / 2}},
$$

where we have introduced the dimensionless function $\beta(\tau, x)$,

$$
\beta(\tau, x)=\left.\frac{1}{4 u_{p}} \frac{\partial}{\partial \tau^{\prime}}\left[\omega \frac{\partial S\left(\tau, \tau^{\prime}\right)}{\partial \tau^{\prime}}-\mathcal{E}\left(\tau^{\prime}\right)\right]\right|_{\tau^{\prime}=\tau-x},
$$

and the quiver radius, $\alpha_{0}=|e| F /\left(m \omega^{2}\right)$, for free-electron oscillations in the field $\mathbf{F}(t)$. The saddle points $x_{s}$ are solutions of the equation

$$
\omega \frac{\partial}{\partial x} S(\tau, \tau-x)+\mathcal{E}(\tau-x)=0 .
$$

The results (31) and (32) for the integral terms $\mathcal{I}^{(0)}$ and $\mathcal{I}_{s}$ allow us to develop an iterative procedure for the solution of Eq. (23) for $f_{\mathbf{p}}(\tau)$ in the low-frequency limit. To do that, we note that the saddle-point contributions, $\mathcal{I}_{s}$, to the integral $\mathcal{I}$ in the approximation (30) are proportional to the dimensional parameter $\alpha_{0}^{-1}=m \omega^{2} /(|e| F)$, while $\mathcal{I}^{(0)}$ is proportional to $\sqrt{2 m \mathcal{E}(\tau)} / \hbar$, where $\mathcal{E}(\tau) \sim u_{p}$. Thus, the ratio of terms $\mathcal{I}_{s}$ to $\mathcal{I}^{(0)}$ is determined by a dimensionless factor $\sim \hbar \omega / u_{p}$. Therefore, the iterative account of terms $\mathcal{I}_{s}$ (which, as we show below, describe the rescattering effects in LAES) is valid when the following condition is satisfied:

$$
\frac{\hbar \omega}{u_{p}} \ll 1 .
$$

It is worthwhile to emphasize that, besides the frequency, the condition (35) involves also the field amplitude $F$, so that the low-frequency expansion for the QES $\Phi_{\mathbf{p}}(\mathbf{r}, t)$ can be called also a "strong-field" expansion, since already for $\hbar \omega / u_{p} \lesssim 1$ the perturbation theory (in laser-atom interaction) for $\Phi_{\mathbf{p}}(\mathbf{r}, t)$ becomes divergent [32].

\section{A. The zero-order approximation for $f_{\mathrm{p}}(\tau)$}

To obtain the zero-order approximation in the parameter $\hbar \omega / u_{p}$ for the function $f_{\mathbf{p}}(\tau)\left[f_{\mathbf{p}}(\tau) \approx f_{\mathbf{p}}^{(0)}(\tau)\right]$, we note that the strongly oscillating exponential factor in Eq. (29) is determined by the inhomogeneous term of Eq. (23) [taking into account Eq. (15)], so that

$$
\begin{gathered}
\mathcal{E}(\tau)=\mathcal{E}^{(0)}(\tau)=\frac{\mathbf{P}^{2}(\tau)}{2 m}, \\
f_{\mathbf{p}}^{(0)}(\tau)=g_{\mathbf{p}}^{(0)}(\tau) e^{-i S_{\mathbf{p}}(\tau) / \hbar} .
\end{gathered}
$$

The preexponential factor $g_{\mathbf{p}}^{(0)}(\tau)$ can be obtained from Eq. (23) after substituting there $f_{\mathbf{p}}(\tau) \rightarrow f_{\mathbf{p}}^{(0)}(\tau)$, omitting then the differential term $\left(\sim \omega d g_{\mathbf{p}}^{(0)} / d \tau\right)$ and the saddle-point contributions to the integral (24) [retaining only the first term in Eq. (30), given by Eq. (31)]. The result for $g_{\mathbf{p}}^{(0)}(\tau)$ is thus

$$
g_{\mathbf{p}}^{(0)}(\tau)=\frac{\kappa}{B_{0}\left[\mathcal{E}^{(0)}(\tau)\right]-i P(\tau) / \hbar}=\kappa \mathcal{A}[P(\tau)],
$$

where the second equality, obtained using Eq. (21), gives the amplitude $\mathcal{A}[P(\tau)]$ [cf. Eq. (28)] for laser-free elastic electronatom $s$-wave scattering in the effective range approximation as a function of the time-dependent kinetic momentum $P(\tau)$. 


\section{B. The first-order (rescattering) correction to $f_{\mathrm{p}}^{(0)}(\tau)$}

The first-order iterative correction $f_{\mathbf{p}}^{(1)}$ to the zero-order result $f_{\mathbf{p}}^{(0)}$ satisfies the equation obtained by substituting

$$
f_{\mathbf{p}}(\tau)=f_{\mathbf{p}}^{(0)}(\tau)+f_{\mathbf{p}}^{(1)}(\tau)
$$

into Eq. (23):

$$
\left(B_{0}\left[\mathcal{E}^{(1)}(\tau)\right]-\frac{i}{\hbar} \sqrt{2 m \mathcal{E}^{(1)}(\tau)}\right) f_{\mathbf{p}}^{(1)}(\tau)=\sum_{s} \mathcal{I}_{s}\left[f_{\mathbf{p}}^{(0)}(\tau)\right],
$$

where $f_{\mathbf{p}}^{(1)}$ is taken in the form (29) with $\mathcal{E}(\tau)=\mathcal{E}^{(1)}(\tau)$ and $g_{\mathbf{p}}(\tau)=g_{\mathbf{p}}^{(1)}(\tau)$. In deriving Eq. (40), the differential term in Eq. (23) is evaluated as follows: We neglected the terms involving $d g_{\mathbf{p}}^{(0)} / d \tau$ and $d g_{\mathbf{p}}^{(1)} / d \tau$ and combined the result of taking the derivative of the exponential [see Eq. (29)] with the term involving $B_{0}(\epsilon)$ [see Eq. (21)] to obtain $B_{0}\left[\mathcal{E}^{(1)}(\tau)\right]$. Also, we used Eq. (31) for $\mathcal{I}^{(0)}\left[f_{\mathbf{p}}^{(1)}(\tau)\right]$. The explicit form for $\mathcal{I}_{s}\left[f_{\mathbf{p}}^{(0)}(\tau)\right]$ follows from Eqs. (32) and (33) taking into account Eqs. (36) and (37):

$$
\mathcal{I}_{s}\left[f_{\mathbf{p}}^{(0)}(\tau)\right]=g_{\mathbf{p}}^{(0)}\left(\tau-x_{s}\right) \frac{e^{i \varphi\left(\tau, x_{s}\right)-i S_{\mathbf{p}}(\tau) / \hbar}}{\alpha_{0}\left[x_{s}^{3} \beta\left(\tau, x_{s}\right)\right]^{1 / 2}},
$$

where

$$
\begin{gathered}
\varphi(\tau, x)=\frac{x[\mathbf{p}-\mathbf{q}(\tau, \tau-x)]^{2}}{2 m \hbar \omega}, \\
\beta(\tau, x)=\frac{\omega^{2}}{e^{2} F^{2}}\left\{\frac{e}{\omega} \mathbf{F}(\tau-x) \cdot[\mathbf{q}(\tau, \tau-x)-\mathbf{p}]\right. \\
\left.+\mathbf{Q}^{2}(\tau-x, \tau) / x\right\}, \\
\mathbf{Q}\left(\tau, \tau^{\prime}\right)=\mathbf{q}\left(\tau, \tau^{\prime}\right)-\frac{e}{c} \mathbf{A}(\tau), \\
\mathbf{q}\left(\tau, \tau^{\prime}\right)=\frac{e}{c} \frac{\int_{\tau^{\prime}}^{\tau} \mathbf{A}\left(\tau^{\prime \prime}\right) d \tau^{\prime \prime}}{\tau-\tau^{\prime}}=\frac{e}{\omega} \frac{\mathbf{F}(\tau)-\mathbf{F}\left(\tau^{\prime}\right)}{\tau-\tau^{\prime}} .
\end{gathered}
$$

For the saddle-point equation (34) we have the following explicit expression:

$$
\mathbf{P}^{2}\left(\tau-x_{s}\right)-\mathbf{Q}^{2}\left(\tau-x_{s}, \tau\right)=0 .
$$

One sees from Eq. (41) for the terms $\mathcal{I}_{s}\left[f_{\mathbf{p}}^{(0)}\right]$ on the righthand side of Eq. (40) that the oscillating (exponential) terms of the function $f_{\mathbf{p}}^{(1)}(\tau)$ are partly determined through the phase functions $\varphi\left(\tau, x_{s}\right)$, which depend on the saddle points $x_{s}$. Thus, the desired function $f_{\mathbf{p}}^{(1)}(\tau)$ can be expressed as a sum,

$$
f_{\mathbf{p}}^{(1)}(\tau)=\sum_{s} g_{\mathbf{p}, s}^{(1)}(\tau) e^{i \varphi\left(\tau, x_{s}\right)-i S_{\mathbf{p}}(\tau) / \hbar},
$$

where we have introduced a set of functions $g_{\mathbf{p}, s}^{(1)}$ corresponding to each saddle point $x_{s}$. Substitution of the form (47) for $f_{\mathbf{p}}^{(1)}$ into Eq. (40) gives the following equation for the preexponential functions $g_{\mathbf{p}, s}^{(1)}$ :

$$
\sum_{s} h_{s}(\tau) e^{i \varphi\left(\tau, x_{s}\right)}=0
$$

$$
\begin{aligned}
h_{s}(\tau)= & \left(B_{0}\left[\mathcal{E}_{s}^{(1)}(\tau)\right]-\frac{i}{\hbar} \sqrt{2 m \mathcal{E}_{s}^{(1)}(\tau)}\right) g_{\mathbf{p}, s}^{(1)}(\tau) \\
& -\frac{g_{\mathbf{p}}^{(0)}\left(\tau-x_{s}\right)}{\alpha_{0}\left[x_{s}^{3} \beta\left(\tau, x_{s}\right)\right]^{1 / 2}},
\end{aligned}
$$

where the set of functions $\mathcal{E}_{s}^{(1)}$ replaces $\mathcal{E}^{(1)}$ in Eq. (40). Comparison of the exponential factors in Eq. (47) with that in Eq. (29) gives the following definition for $\mathcal{E}_{s}^{(1)}(\tau)$ :

$$
\begin{aligned}
\mathcal{E}_{s}^{(1)}(\tau) & =\epsilon-\hbar \omega \frac{d}{d \tau}\left[\varphi\left(\tau, x_{s}\right)-S_{\mathbf{p}}(\tau) / \hbar\right] \\
& =\mathbf{Q}^{2}\left(\tau, \tau-x_{s}\right) /(2 m) .
\end{aligned}
$$

In order to proceed we assume that any two different solutions, $x_{s}$ and $x_{s^{\prime}}$, of Eq. (46) do not merge with variation of $\tau$ and, moreover, are such that the inequality

$$
\left|\frac{d}{d \tau}\left[\varphi\left(\tau, x_{s}\right)-\varphi\left(\tau, x_{s^{\prime}}\right)\right]\right| \gtrsim u_{p} /(\hbar \omega)
$$

is fulfilled for the range of values of $\mathbf{p}$ and parameters of the field $\mathbf{F}(t)$ considered in this paper. [The validity of Eq. (51) can be justified by a numerical analysis of Eq. (46) (cf. Sec. V A).] Under this assumption, the exponential factors in Eq. (48) can be considered as quasiorthogonal functions in the following sense:

$$
\left|\int h_{s}(\tau) e^{i\left[\varphi\left(\tau, x_{s}\right)-\varphi\left(\tau, x_{s^{\prime}}\right)\right]} d \tau\right| \ll\left|\int h_{s}(\tau) d \tau\right|, \quad s \neq s^{\prime} .
$$

Therefore, without losing accuracy, we can consider only the trivial solution of Eq. (48), $h_{s}(\tau)=0$, which from Eq. (49) gives a set of uncoupled equations for the functions $g_{\mathbf{p}, s}^{(1)}(\tau)$.

Finally, taking into account Eqs. (38) and (50), the preexponential factors $g_{\mathbf{p}, s}^{(1)}(\tau)$, which determine the first-order correction $f_{\mathbf{p}}^{(1)}(\tau)$ in Eq. (47), can be expressed in terms of two field-free elastic scattering amplitudes (28) with different, field-dependent momenta:

$$
g_{\mathbf{p}, s}^{(1)}(\tau)=\frac{\kappa \mathcal{A}\left[P\left(\tau-x_{s}\right)\right] \mathcal{A}\left[Q\left(\tau, \tau-x_{s}\right)\right]}{\alpha_{0} \sqrt{x_{s}^{3} \beta\left(\tau, x_{s}\right)}} .
$$

The most remarkable consequences of Eqs. (38) and (52) are that (i) both results involve an exact (within effective range theory), non-Born field-free scattering amplitude $\mathcal{A}(p)$ with laser-modified momentum and (ii) the result (52) involves this amplitude twice. Fact (ii) allows us to call the approximate result (47) "the rescattering approximation." Thus, the existence of laser-induced recollisions in laser-assisted collision processes becomes apparent already on the level of the QES wave function $\Phi_{\mathbf{p}}(\mathbf{r}, t)$, in which the electron-atom dynamics and its modification by a strong laser field are completely described by the function $f_{\mathbf{p}}(t)$. The low-frequency analysis of the exact TDER equation (23), presented in this section, allows us to obtain analytic closed-form expressions for the LAES amplitude (26) corresponding to the zero-order [Eqs. (37) and (38)] and rescattering [Eqs. (47) and (52)] approximations for $f_{\mathbf{p}}(\tau)$ and, therefore, for the scattering state $\Phi_{\mathbf{p}}(\mathbf{r}, t)$. 


\section{THE ZERO-ORDER (KROLL-WATSON) APPROXIMATION FOR THE LAES CROSS SECTION}

Using the zero-order approximation $f_{\mathbf{p}}(\tau) \approx f_{\mathbf{p}}^{(0)}(\tau)$ [where $f_{\mathbf{p}}^{(0)}(\tau)$ is given by Eqs. (37) and (38)], we obtain for the LAES amplitude (26) the expression

$$
\mathcal{A}_{n}^{(0)}\left(\mathbf{p}, \mathbf{p}_{n}\right)=\frac{1}{2 \pi} \int_{0}^{2 \pi} \mathcal{A}[P(\tau)] e^{i n \tau+i \Delta_{n}(\tau)} d \tau,
$$

where

$$
\begin{aligned}
\Delta_{n}(\tau) & =\left[S_{\mathbf{p}_{n}}(\tau)-S_{\mathbf{p}}(\tau)\right] / \hbar=\rho \cos \left(\tau-\varphi_{\mathbf{t}}\right), \\
\rho & =\frac{|e| F}{m \hbar \omega^{2}}|\mathbf{e} \cdot \mathbf{t}|, \quad \varphi_{\mathbf{t}}=\arg (\mathbf{e} \cdot \mathbf{t}), \quad \mathbf{t}=\mathbf{p}_{n}-\mathbf{p},
\end{aligned}
$$

and the scalar product $(\mathbf{e} \cdot \mathbf{t})$ is defined in accordance with Eq. (5). For the more general case of $l$-wave scattering, a low-frequency analysis of the TDER equations leads to the expression (53) for the scattering amplitude in which $\mathcal{A}[P(\tau)]$ is replaced by $\mathcal{A}^{(l)}\left[\mathbf{P}(\tau), \mathbf{P}_{n}(\tau)\right]$, where $\mathbf{P}_{n}(\tau)=$ $\mathbf{p}_{n}-(e / c) \mathbf{A}(\tau)$,

$$
\mathcal{A}^{(l)}\left(\mathbf{p}_{i}, \mathbf{p}_{f}\right)=\frac{(2 l+1)\left(k_{i} k_{f}\right)^{l} \mathcal{P}_{l}\left(\cos \theta_{\mathrm{if}}\right)}{-1 / a_{l}+r_{l} k_{i}^{2} / 2-i k_{i}^{2 l+1}},
$$

$k_{i, f}=\left|\mathbf{p}_{i, f}\right| / \hbar, \mathcal{P}_{l}(x)$ is a Legendre polynomial, and $\theta_{\text {if }}=$ $\angle\left(\mathbf{p}_{i}, \mathbf{p}_{f}\right)$. Later, we omit the superscript $(l)$ denoting the amplitude for field-free scattering (54), $\mathcal{A}\left(\mathbf{p}_{i}, \mathbf{p}_{f}\right) \equiv \mathcal{A}^{(l)}\left(\mathbf{p}_{i}, \mathbf{p}_{f}\right)$, bearing in mind that $\mathcal{A}\left(\mathbf{p}_{i}, \mathbf{p}_{f}\right)$ contains information about the spatial symmetry of the bound state supported by the scattering potential. Note that the amplitude $\mathcal{A}^{(s)}(p)$ for elastic $s$-wave scattering in Eq. (28) is isotropic and depends only on the modulus of the initial momentum. Thus, if necessary, the difference between $\mathcal{A}^{(s)}(p)$ and $\mathcal{A}^{(l \neq 0)}\left(\mathbf{p}_{i}, \mathbf{p}_{f}\right)$ will be indicated by using a different number of arguments.

It is important to note that the "instantaneous" amplitude $\mathcal{A}\left[\mathbf{P}(\tau), \mathbf{P}_{n}(\tau)\right]$ that replaces $\mathcal{A}[P(\tau)]$ in Eq. (53) is not an elastic scattering amplitude (since $|\mathbf{P}(\tau)| \neq\left|\mathbf{P}_{n}(\tau)\right|$ ). For the case of linear polarization $(\ell=1) \mathrm{Eq}$. (53) corresponds to Eq. (5.16) in Ref. [15], which involves the $T$ matrix off the energy shell. For the case of elliptical polarization, results identical to Eq. (53) were obtained in Refs. [33,34].

In the low-frequency limit $(\rho \gg 1)$, the amplitude (53) can be evaluated analytically using uniform asymptotic approximation methods for integrals [35,36] (cf. Appendix B):

$$
\mathcal{A}_{n}^{(0)}=i^{n} e^{i n \varphi_{\mathrm{t}}}\left[\mathcal{A}_{+} J_{n}(\rho)+\mathcal{A}_{-} \frac{i \rho J_{n}^{\prime}(\rho)}{\sqrt{\rho^{2}-n^{2}}}\right],
$$

where $J_{n}^{\prime}(x)$ is the derivative of the Bessel function $J_{n}(x)$,

$$
\mathcal{A}_{ \pm}=\frac{1}{2}\left[\mathcal{A}_{\mathrm{el}}\left(\tau_{+}\right) \pm \mathcal{A}_{\mathrm{el}}\left(\tau_{-}\right)\right]
$$

and $\mathcal{A}_{\mathrm{el}}\left(\tau_{ \pm}\right) \equiv \mathcal{A}\left[\mathbf{P}\left(\tau_{ \pm}\right), \mathbf{P}_{n}\left(\tau_{ \pm}\right)\right]$, where $\tau=\tau_{ \pm}$are saddle points of the integrand in Eq. (53) that satisfy the equation

$$
\rho \sin \left(\tau-\varphi_{\mathbf{t}}\right)=n \text {. }
$$

Because of the equality $\left|\mathbf{P}\left(\tau_{ \pm}\right)\right|=\left|\mathbf{P}_{n}\left(\tau_{ \pm}\right)\right|, \mathcal{A}_{\mathrm{el}}\left(\tau_{ \pm}\right)$is the on-shell amplitude for elastic field-free scattering with lasermodified momenta. This modification serves to displace $\mathbf{p}$ and $\mathbf{p}_{n}$ by the shift $\Delta \mathbf{p}_{ \pm}=(|e| / c) \mathbf{A}\left(\tau_{ \pm}\right)$. For the classically allowed region $|n| \leqslant \rho$, Eq. (57) gives

$$
\begin{gathered}
\tau_{ \pm}=\varphi_{\mathbf{t}}+\frac{\pi}{2} \pm \arccos \frac{n}{\rho}, \\
\Delta \mathbf{p}_{ \pm}=-\frac{m \hbar \omega}{2|\mathbf{e} \cdot \mathbf{t}|^{2}}\left\{ \pm \xi[\hat{\boldsymbol{\kappa}} \times \mathbf{t}] \sqrt{\rho^{2}-n^{2}}\right. \\
+n[2 \ell(\hat{\boldsymbol{\epsilon}} \cdot \mathbf{t}) \hat{\boldsymbol{\epsilon}}+(1-\ell)(\mathbf{t}-(\hat{\boldsymbol{\kappa}} \cdot \mathbf{t}) \hat{\boldsymbol{\kappa}})]\},
\end{gathered}
$$

where the degrees of linear $(\ell)$ and circular $(\xi)$ polarization are defined in Eq. (4). Note that for the case of critical geometry, when $(\mathbf{e} \cdot \mathbf{t}) \rightarrow 0$ (and thus $\rho \approx 0$ ), the result (55), based on a saddle-point analysis of the integral (53), is not applicable.

The result (55) for $\mathcal{A}_{n}^{(0)}$ and the corresponding cross section,

$$
\frac{d \sigma_{n}^{(0)}\left(\mathbf{p}, \mathbf{p}_{n}\right)}{d \Omega_{\mathbf{p}_{n}}}=\frac{p_{n}}{p}\left|\mathcal{A}_{+} J_{n}(\rho)+\mathcal{A}_{-} \frac{i \rho J_{n}^{\prime}(\rho)}{\sqrt{\rho^{2}-n^{2}}}\right|^{2},
$$

may be simplified and reduced to the well-known KrollWatson formula [15] for the following particular cases of the laser polarization and the scattering geometry:

(i) For the case of linear polarization $(\ell=1)$, we have $\Delta \mathbf{p}_{+}=\Delta \mathbf{p}_{-}=\Delta \mathbf{p}$, where

$$
\Delta \mathbf{p}=-m \hbar \omega n \frac{\hat{\boldsymbol{\epsilon}}}{(\hat{\boldsymbol{\epsilon}} \cdot \mathbf{t})},
$$

so that $\mathcal{A}_{+}=\mathcal{A}_{\mathrm{el}}\left(\mathbf{p}+\Delta \mathbf{p}, \mathbf{p}_{n}+\Delta \mathbf{p}\right)$ and $\mathcal{A}_{-}=0$, while the cross section (60) reduces to the original Kroll-Watson result [15]:

$$
\frac{d \sigma_{n}^{(\mathrm{KW})}\left(\mathbf{p}, \mathbf{p}_{n}\right)}{d \Omega_{\mathbf{p}_{n}}}=\frac{p_{n}}{p} J_{n}^{2}(\rho) \frac{d \sigma_{\mathrm{el}}\left(\mathbf{P}, \mathbf{P}_{n}\right)}{d \Omega_{\mathbf{P}_{n}}},
$$

where $d \sigma_{\mathrm{el}} / d \Omega=\left|\mathcal{A}_{\mathrm{el}}\right|^{2}$ is the exact cross section for field-free elastic scattering and $\mathbf{P} \equiv \mathbf{p}+\Delta \mathbf{p}, \mathbf{P}_{n} \equiv \mathbf{p}_{n}+\Delta \mathbf{p}$. Note that the momentum shift $\Delta \mathbf{p}$ for the case of linear polarization remains real in the classically forbidden region $|n|>\rho$.

(ii) For the cases of forward and backward scattering along the major axis of the polarization ellipse $\left(\mathbf{p}\left\|\mathbf{p}_{n}\right\| \hat{\boldsymbol{\epsilon}}\right), \Delta \mathbf{p}_{ \pm}$in Eq. (59) reduces as follows:

$$
\Delta \mathbf{p}_{ \pm}=-\frac{m \hbar \omega}{|\mathbf{t}|}\left[\hat{\boldsymbol{\epsilon}} n \pm \frac{\xi}{1+\ell}[\hat{\boldsymbol{\kappa}} \times \hat{\boldsymbol{\epsilon}}] \sqrt{\rho^{2}-n^{2}}\right]
$$

The collinearity of the vectors $\mathbf{p}, \mathbf{p}_{n}$, and $\mathbf{t}$ gives the following relations: $\left|\mathbf{P}\left(\tau_{+}\right)\right|=\left|\mathbf{P}\left(\tau_{-}\right)\right|, \mathbf{P}\left(\tau_{ \pm}\right)=-\mathbf{P}_{n}\left(\tau_{\mp}\right)$. Thus, $\mathcal{A}_{-}=0$, $\mathcal{A}_{+}=\mathcal{A}_{\mathrm{el}}\left(\tau_{+}\right)=\mathcal{A}_{\mathrm{el}}\left(\tau_{-}\right)$, and the LAES cross section is given by Eq. (61), with $\mathbf{P}=\mathbf{p}+\Delta \mathbf{p}_{ \pm}$and $\mathbf{P}_{n}=\mathbf{p}_{n}+\Delta \mathbf{p}_{ \pm}$, where $\Delta \mathbf{p}_{ \pm}$is given by Eq. (62). (This result is the same using either $\Delta \mathbf{p}_{+}$or $\left.\Delta \mathbf{p}_{-}.\right)$

(iii) For forward or backward scattering in the polarization plane for a circularly polarized field $(\ell=0)$, Eq. (59) gives

$$
\Delta \mathbf{p}_{ \pm}=-\frac{m \hbar \omega}{|\mathbf{t}|^{2}}\left[\mathbf{t} n \pm \xi[\hat{\boldsymbol{\kappa}} \times \mathbf{t}] \sqrt{\rho^{2}-n^{2}}\right] .
$$

With $\Delta \mathbf{p}_{ \pm}$given by Eq. (63), the same analysis as for case (ii) is then valid.

Note that other analytic expressions for the scattering amplitude (53) were obtained in Refs. [33,34]. The LAES amplitude in the low-frequency approximation introduced by Madsen and Taulbjerg [34] [labeled the "peaked impulse approximation" (PIA)] has a form similar to Eq. (55), but involves the Anger and Weber functions [37] [cf. Eq. (B7) in Appendix B]. In Fig. 1 we compare the PIA result of Ref. [34] 


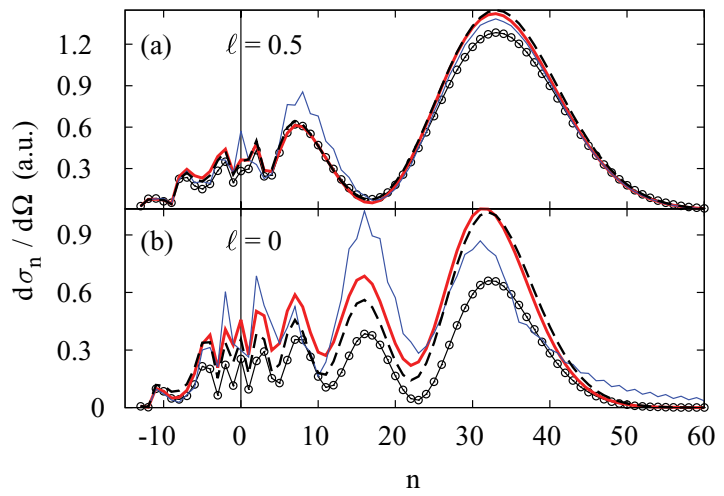

FIG. 1. (Color online) Differential cross section $d \sigma_{n}\left(\mathbf{p}, \mathbf{p}_{n}\right) / d \Omega_{\mathbf{p}_{n}}$ for laser-assisted $s$-wave $e$-H scattering in the polarization plane $(\mathbf{p} \| \hat{\boldsymbol{\epsilon}}$, $\left.\mathbf{p}_{n} \perp \hat{\boldsymbol{\kappa}}\right)$ for a scattering angle $\boldsymbol{\theta} \equiv \angle\left(\mathbf{p}, \mathbf{p}_{n}\right)=20^{\circ}$ in a $\mathrm{CO}_{2}$-laser field with $\hbar \omega=0.117 \mathrm{eV}(\lambda=10.6 \mu \mathrm{m})$ and intensity $I=2.5 \times$ $10^{11} \mathrm{~W} / \mathrm{cm}^{2}$. The incident electron energy is $E=1.58 \mathrm{eV}$ and $n$ is the number of photons absorbed $(n>0)$ or emitted $(n<0)$. Results are shown for two laser polarizations: (a) elliptical polarization, with $\eta=+0.58(\ell=0.5)$; (b) circular polarization, with $\eta=+1$ $(\ell=0)$. Circles, exact TDER results [cf. Eqs. (A6), (27)]; dashed lines, results using the approximate amplitude (53); thick solid (red) lines, Eq. (60); thin solid (blue) lines, peaked impulse approximation (PIA) result of Ref. [34].

with the analytic result (55), the integral expression (53) (within the effective range approximation), and the exact TDER results. The effective range theory parameters are those for $e-\mathrm{H}$ scattering: $\left|E_{0}\right|=0.755 \mathrm{eV}, \kappa=0.236$ a.u., $a_{0}=1.453 \kappa^{-1}$, and $r_{0}=0.623 \kappa^{-1}$. One sees in Fig. 1 that the zero-order approximation (53) for the LAES amplitude reproduces well the oscillation pattern in the LAES spectrum. It follows from Eq. (55) that these oscillations are well approximated by the Bessel function and its derivative; they originate from an interference of two classical electron trajectories corresponding to two different times of collision, $\tau_{+}$and $\tau_{-}$. In contrast, the result of Ref. [34] exhibits an additional sharp oscillatory structure for $d \sigma_{n} / d \Omega$ as a function of $n$ that stems from properties of the Weber function; they do not have any physical meaning.

As may be seen from Eq. (58), the two real saddle points $\tau_{ \pm}$coalesce at the cutoff of the classically allowed region (i.e., for $n=\rho$ ). In the classically forbidden region $(|n|>\rho)$, the solutions of the saddle-point equation (57) and the corresponding momentum shifts (59) become complex, so we analytically continue the result (55) to this case. However, the complex displacements of momenta in the elastic scattering amplitude may cause some nonphysical features in the LAES cross section. Thus, for example, for electron scattering with absorption or emission of $|n|>\rho \gg 1$ laser photons, the condition $\mathbf{P}^{2}\left(\tau_{ \pm}\right) /(2 m)=-\left|E_{0}\right|$ may be satisfied for appropriate laser parameters and geometry of the incident and outgoing electrons. For such conditions, the amplitude $\mathcal{A}_{\mathrm{el}}$ has a pole, which corresponds to some point $\tau=\tau^{(p)}$ (or to more than one point) on the complex plain of $\tau$. The coalescence of one of the saddle points $\tau_{ \pm}$with the point $\tau^{(p)}$ leads to the appearance of a nonphysical resonantlike enhancement of the LAES cross section. (This fact is exhibited most clearly for the case of forward scattering and circular polarization.)

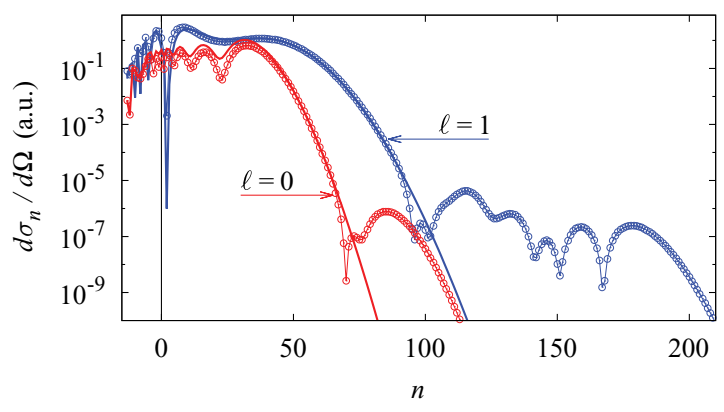

FIG. 2. (Color online) The same as in Fig. 1, but for the cases of linear $(\ell=1)$ and circular $(\ell=0)$ polarization and for a larger range of $n>0$. Circles, exact TDER results [cf. Eqs. (A6) and (27)]; solid lines, results using the analytic amplitude (55).

Thus, for the general case of elliptical polarization, the result (55) has limited applicability in the classically forbidden region. For this case, an alternative analytic result, suggested in Ref. [34], is obtained within an additional weak-field approximation and, therefore, is not applicable for the description of strong laser field effects, such as the plateau structures in LAES spectra.

In Fig. 2 we present LAES spectra for $e$-H scattering in linearly and circularly polarized $\mathrm{CO}_{2}$-laser fields. The field intensity, electron energy, and scattering geometry are the same as in Fig. 1. For both of these two limiting cases of the laser polarization, $\ell=1$ and $\ell=0$, as well as for the general case of elliptical polarization $(0 \leqslant \ell \leqslant 1)$, the zero-order (Kroll-Watson) approximation (55) for the LAES-amplitude does not describe the high-energy part of the LAES spectra (i.e., the rescattering plateau), for which a proper account of laser-induced electron rescattering from the potential $U(r)$ is required $[9,10]$. For the low-energy plateau, the result (55) is in good agreement with the exact TDER results, except for the case of the critical geometry [for which $\mathbf{e} \cdot\left(\mathbf{p}_{n}-\mathbf{p}\right)=0$ ], as exhibited, for example, by the pronounced suppression of the LAES cross section within the Kroll-Watson approximation as compared to the exact result for $n=2$ and $\ell=1$ (cf. Fig. 2). This discrepancy is due to the fact that the scattering angle $\theta=20^{\circ}$ is close to the critical angle, $\theta_{\mathrm{cr}}=21.05^{\circ}$, for the channel $n=2$.

\section{THE RESCATTERING APPROXIMATION FOR THE LAES AMPLITUDE}

The rescattering correction $\mathcal{A}_{n}^{(1)}$ to the zero-order result (55) for the LAES amplitude,

$$
\mathcal{A}_{n}\left(\mathbf{p}, \mathbf{p}_{n}\right) \approx \mathcal{A}_{n}^{(0)}+\mathcal{A}_{n}^{(1)},
$$

follows upon substituting $f_{\mathbf{p}}(\tau)=f_{\mathbf{p}}^{(1)}(\tau)$ [where $f_{\mathbf{p}}^{(1)}(\tau)$ is given by Eqs. (47) and (52)] into Eq. (26) to obtain

$$
\begin{aligned}
& \mathcal{A}_{n}^{(1)}=\sum_{s} \mathcal{A}_{n, s}^{(1)}, \\
& \mathcal{A}_{n, s}^{(1)}=\frac{1}{2 \pi \alpha_{0}} \int_{0}^{2 \pi} \mathcal{A}\left[P\left(\tau_{s}^{\prime}\right)\right] \mathcal{A}\left[Q\left(\tau, \tau_{s}^{\prime}\right)\right] \frac{e^{i \phi_{s}(\tau)} d \tau}{\sqrt{x_{s}^{3} \beta\left(\tau, x_{s}\right)}},
\end{aligned}
$$

where we have defined $\tau_{s}^{\prime} \equiv \tau-x_{s}$ and, using Eq. (15),

$$
\phi_{s}(\tau) \equiv \varphi\left(\tau, x_{s}\right)+n \tau-\frac{e}{m \hbar \omega^{2}}\left(\mathbf{p}_{n}-\mathbf{p}\right) \cdot \mathbf{F}(\tau),
$$


where the functions $\varphi(\tau, x)$ and $\beta(\tau, x)$ are defined in Eqs. (42) and (43), respectively, and $x_{s}=x_{s}(\tau)$ is defined implicitly by Eq. (46).

For the case of $l$-wave scattering, our analysis of the rescattering correction to the LAES amplitude yields again an expression like (65), but with the field-free $s$-wave scattering amplitude $\mathcal{A}(p)$ [cf. Eq. (28)] in the integrand of (65) replaced by $\mathcal{A}\left(\mathbf{p}_{i}, \mathbf{p}_{f}\right)$ [cf. Eq. (54)]:

$$
\begin{aligned}
\mathcal{A}\left[P\left(\tau_{s}^{\prime}\right)\right] & \rightarrow \mathcal{A}\left[\mathbf{P}\left(\tau_{s}^{\prime}\right), \mathbf{Q}\left(\tau_{s}^{\prime}, \tau\right)\right], \\
\mathcal{A}\left[Q\left(\tau, \tau_{s}^{\prime}\right)\right] & \rightarrow \mathcal{A}\left[\mathbf{Q}\left(\tau, \tau_{s}^{\prime}\right), \mathbf{P}_{n}(\tau)\right],
\end{aligned}
$$

where $\mathbf{Q}\left(\tau, \tau^{\prime}\right)$ is defined by Eqs. (44) and (45).

The dominant contributions to the integral (65) come from the vicinity of the saddle points $\tau=\tau_{k}$, which satisfy the equation

$$
\left.2 m \hbar \omega \frac{d \phi_{s}}{d \tau}\right|_{\tau=\tau_{k}}=\mathbf{P}_{n}^{2}\left(\tau_{k}\right)-\mathbf{Q}^{2}\left(\tau_{k}, \tau_{k}-x_{s}\right)=0 .
$$

[In deriving Eq. (67), use has been made of the relations $n \hbar \omega=\left(\mathbf{p}_{n}^{2}-\mathbf{p}^{2}\right) /(2 m), d \mathbf{F} / d \tau=(\omega / c) \mathbf{A}(\tau)$, and Eq. (46).] The saddle-point equations (46) and (67) comprise a system of coupled equations having a transparent physical meaning. Upon colliding with an atom at the time moment $\tau_{s, k}^{\prime}=\tau_{k}-x_{s}$, the electron changes its momentum $\mathbf{p}$ to a field-dependent "intermediate" momentum $\mathbf{q}\left(\tau_{k}, \tau_{s, k}^{\prime}\right)$, which ensures the condition for return of the electron by the laser field back to the atom at the time moment $\tau_{k}$ followed by a rescattering. The set of points $x_{s}$ determines the excursion times of the returning electron along different closed classical trajectories, while Eqs. (46) and (67) represent the energy conservation laws at the times of the first and second collisions. The argument $\mathbf{Q}$ of the field-free amplitude $\mathcal{A}$ in Eq. (65) is the instantaneous kinetic momentum of the electron in the laser field in the "intermediate" state with canonical momentum q [cf. Eq. (44)].

\section{A. Analysis of the saddle-point equations}

To evaluate the integral in Eq. (65), it is instructive to analyze first the solutions of the system of coupled saddle-point equations (46) and (67). Using dimensionless quantities, this system may be represented as follows:

$$
\begin{aligned}
& \boldsymbol{\gamma}^{2}-\boldsymbol{v}^{2}+2(\boldsymbol{\gamma}-\boldsymbol{v}) \cdot \operatorname{Im}\left(\mathbf{e} e^{-i \tau^{\prime}}\right)=0, \\
& \boldsymbol{\gamma}_{n}^{2}-\boldsymbol{v}^{2}+2\left(\boldsymbol{\gamma}_{n}-\boldsymbol{v}\right) \cdot \operatorname{Im}\left(\mathbf{e} e^{-i \tau}\right)=0,
\end{aligned}
$$

where $\boldsymbol{\gamma} \equiv \omega \mathbf{p} /(|e| F), \boldsymbol{\gamma}_{n} \equiv \omega \mathbf{p}_{n} /(|e| F)$, and $\boldsymbol{v} \equiv \boldsymbol{v}\left(\tau, \tau^{\prime}\right)=$ $\omega \mathbf{q}\left(\tau, \tau^{\prime}\right) /(|e| F)$.

Despite the fact that Eqs. (68) and (69) are very similar, their solutions in the plane of variables $\tau$ and $\tau^{\prime}$ (or, alternatively, $\tau$ and $x$, where $x=\tau-\tau^{\prime}$ ) differ because of the different ranges of the parameters $\gamma$ and $\gamma_{n}$. Indeed, rescattering effects become important in the region of the LAES spectrum where "direct" scattering is classically forbidden, that is, beyond the region of validity of the Kroll-Watson result, where $\gamma_{n}>\sqrt{2(1+\ell)}-$ $\gamma\left(\right.$ for $\left.\boldsymbol{\gamma}_{n}\|\boldsymbol{\gamma}\| \hat{\boldsymbol{\epsilon}}\right)[11,38]$. On the other hand, rescattering effects are most pronounced for low incident electron energy, that is, $E \lesssim 2 u_{p}$ or $\gamma \lesssim 1$.

The numerical solutions of Eq. (68) for $\gamma=0.6(\boldsymbol{\gamma} \| \hat{\boldsymbol{\epsilon}})$ and Eq. (69) for different values of $\gamma_{n}\left(\boldsymbol{\gamma}_{n} \| \hat{\boldsymbol{\epsilon}}\right)$ for the case of elliptical polarization with $\eta=0.5$ is shown in Fig. 3(a).
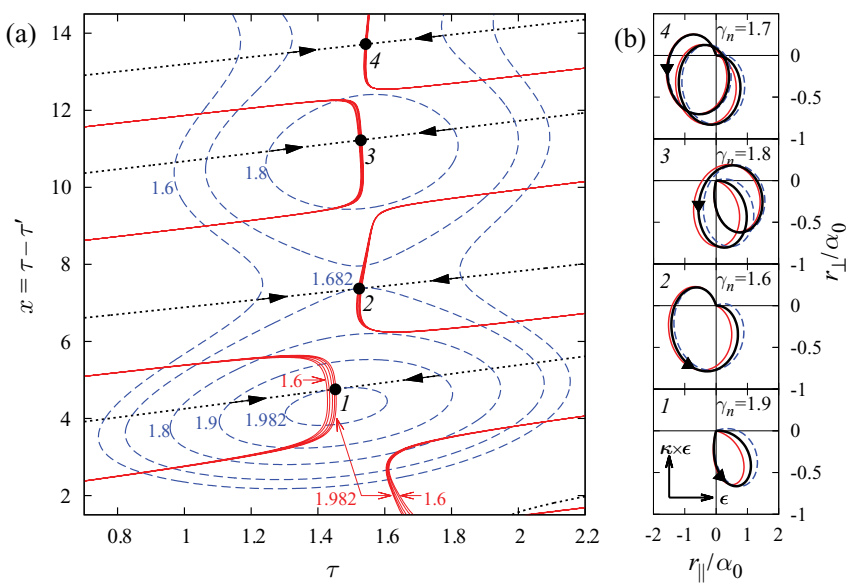

FIG. 3. (Color online) (a) The solutions of Eqs. (68) (dotted lines), (69) (dashed lines), and (70) (solid lines) for different values of $\gamma_{n}$, indicated in the figure near the corresponding curve, $\gamma=0.6$, $\boldsymbol{\gamma}\left\|\boldsymbol{\gamma}_{n}\right\| \hat{\boldsymbol{\epsilon}}$, and polarization $\eta=0.5$. The black arrows show the direction of movement of the coalescing solutions of the coupled equations system (68) and (69) with increasing $\gamma_{n}$. The corresponding coalescence points [the solutions of the system of Eqs. (68) and (70)] are indicated by the black dots labeled by the numerals $s=1,2,3,4$. (b) The classical closed trajectories of the electron in the polarization plane of the field $\mathbf{F}(t)$. Thick solid (black) line, the coalesced (extremal) trajectories corresponding to the solutions $s=1,2,3,4$ of Eqs. (68) and (70); thin solid (red) and thin dashed (blue) lines, the short and long coalescing trajectories corresponding to the solutions of Eqs. (68) and (69).

Figure 3(a) illustrates the fact that, for the range of parameters considered, Eq. (69) has at most two real solutions $\tau_{ \pm}^{(s)}$ on the trajectory $x=x_{s}(\tau)$ of each solution of Eq. (68). With increasing $\gamma_{n}$, the points $\tau_{ \pm}^{(s)}$ tend toward each other and coalesce at $\tau=\tau_{s}$ for $\gamma_{n}=\gamma_{n}^{(s)}$. For example, the point 1 $\left(\tau_{1}=1.453, x_{1}=4.764\right)$ in Fig. 3 corresponds to $\gamma_{n}=\gamma_{n}^{(1)}=$ 1.982 , while the point $2\left(\tau_{2}=1.523, x_{2}=7.368\right)$ corresponds to $\gamma_{n}^{(2)}=1.682$.

The coalescence of two real solutions, $\tau_{+}^{(s)}=\tau_{-}^{(s)}=\tau_{s}$, at $\gamma_{n}=\gamma_{n}^{(s)}$ and their disappearance for $\gamma_{n}>\gamma_{n}^{(s)}$ means that the first derivative of $\phi_{s}(\tau)$ has a local minimum at $\tau=\tau_{s}$, while $\tau$ and $x$ vary along the trajectory of the solution $x=x_{s}(\tau)$. Thus, the point $\tau=\tau_{s}, x=x_{s} \equiv x_{s}\left(\tau_{s}\right)$ satisfies two coupled equations: Eq. (68) and $d^{2} \phi_{s} / d \tau^{2}=0$. The latter equation may be written as

$\left(\boldsymbol{v}-\boldsymbol{\gamma}_{n}\right) \cdot \operatorname{Re}\left(\mathbf{e} e^{-i \tau}\right)\left(\tau-\tau^{\prime}\right)+\mathcal{Q}^{2}-\mathcal{Q} \cdot \mathcal{Q}^{\prime} \frac{d \tau^{\prime}}{d \tau}=0$,

where the notations

$$
\begin{aligned}
& \mathcal{Q}=\boldsymbol{v}\left(\tau, \tau^{\prime}\right)+\operatorname{Im}\left(\mathbf{e} e^{-i \tau}\right), \\
& \mathcal{Q}^{\prime}=\boldsymbol{v}\left(\tau, \tau^{\prime}\right)+\operatorname{Im}\left(\mathbf{e} e^{-i \tau^{\prime}}\right),
\end{aligned}
$$

have been used and where $d \tau^{\prime} / d \tau$ is determined implicitly by Eq. (68):

$$
\frac{d \tau^{\prime}}{d \tau}=\frac{\mathcal{Q} \cdot \mathcal{Q}^{\prime}}{(\boldsymbol{\gamma}-\boldsymbol{v}) \cdot \operatorname{Re}\left(\mathbf{e} e^{-i \tau^{\prime}}\right)\left(\tau-\tau^{\prime}\right)+\mathcal{Q}^{\prime 2}} .
$$

As one sees in Fig. 3(a), the solution $\left(\tau_{s}, x_{s}\right)$ of the system of Eqs. (68) and (70) depends only weakly on $\gamma_{n}$. 
The solutions $\left(\tau_{s}, x_{s}\right)$ may be grouped in pairs, labeled by two consecutive (odd and even) integer subscripts $s$ [with the solutions $\left(\tau_{s}, x_{s}\right)$ enumerated in order of increasing values of $x_{s}$, starting with $\left.s=1\right]$. Analysis of the system of Eqs. (68) and (70) shows that the odd- and even-numbered solutions of each pair correspond, respectively, to greater and smaller values of $\gamma_{n}^{(s)}$. Moreover, the first pair of solutions (i.e., $s=1,2$ ) provide two limiting values for $\gamma_{n}^{(s)}$ : For $\gamma_{n}>\gamma_{n}^{(1)} \equiv \gamma_{n}$, max the system (68) and (69) does not have real solutions [the derivative $d \phi_{s}(\tau) / d \tau$ as a function of $\tau$ and $s$ has a global minimum at the point $\left.\left(\tau_{1}, x_{1}\right)\right]$, while the two saddle points $\tau_{ \pm}^{(s)}$ do not coalesce for $\gamma_{n}<\gamma_{n}^{(2)}$. All other solutions $\left(\tau_{s}, x_{s}\right)$ correspond to intermediate values of $\gamma_{n}^{(s)}$. A similar alternation of $\gamma_{n}^{(s)}$ with increasing $x_{s}$ exists also in the analysis of the ATI process and was described within the semiclassical rescattering model in Ref. [2].

Considering the classical motion of the electron in the laser field $\mathbf{F}(t)$ described by Newton's equation, $m \ddot{\mathbf{r}}=-e \mathbf{F}(t)$, a closed classical trajectory may be found for each solution of the saddle-point equations (68) and (69). For the geometry $\boldsymbol{\gamma}_{n}\|\boldsymbol{\gamma}\| \hat{\boldsymbol{\epsilon}}$ and an elliptically polarized laser field, these trajectories lie in the polarization plane $\left(\mathbf{r}=r_{\|} \hat{\boldsymbol{\epsilon}}+r_{\perp}[\hat{\boldsymbol{\kappa}} \times \hat{\boldsymbol{\epsilon}}]\right)$ and are shown in Fig. 3(b) for different values $\gamma_{n}$. The two different rescattering times, $\tau_{+}^{(s)}$ and $\tau_{-}^{(s)}$, correspond to the long and short trajectories, respectively, while the coalescence point $\left(\tau_{s}, x_{s}\right)$ corresponds to the extreme trajectory with $\gamma_{n}=\gamma_{n}^{(s)}$. The smallest value of $x_{s}$ (i.e., $x_{1}$ ) is the return time of the electron along the shortest extreme closed path. During its motion along this shortest trajectory, the electron gains the maximal classical kinetic energy $E_{n, \max }=2 u_{p} \gamma_{n, \max }^{2}$.

With increasing $s$ (for $x_{s} \gg 1$ ), the solutions $\tau_{s}$ tend to a constant value (independent of $s$ ), while the sets of solutions $x_{s}$ with odd and even $s$ become equidistant: $\left(x_{s+2}-x_{s}\right) \rightarrow$ $2 \pi$. This fact is easily verified by considering the solution of Eqs. (68) and (70) in the limit $x=\tau-\tau^{\prime} \gg 1$. For this case, assuming $|\boldsymbol{\gamma} \cdot \mathbf{e}| \neq 0$ and $\left|\boldsymbol{\gamma}_{n} \cdot \mathbf{e}\right| \neq 0$, the system (68) and (70) reduces to the much simpler system

$$
\boldsymbol{\gamma}^{2}+2 \boldsymbol{\gamma} \cdot \operatorname{Im}\left(\mathbf{e} e^{-i(\tau-x)}\right)=0, \quad \boldsymbol{\gamma}_{n} \cdot \operatorname{Re}\left(\mathbf{e} e^{-i \tau}\right)=0,
$$

which has the following solution:

$$
\begin{gathered}
\tau=\varphi_{\gamma_{n}}+\frac{\pi}{2}, \\
x_{2 k-(1 \pm 1) / 2}=\varphi_{\gamma_{n}}-\varphi_{\boldsymbol{\gamma}}+2 \pi k \pm \arccos \frac{\boldsymbol{\gamma}^{2}}{2|\boldsymbol{\gamma} \cdot \mathbf{e}|},
\end{gathered}
$$

where $\varphi_{\gamma_{n}}=\arg \left(\boldsymbol{\gamma}_{n} \cdot \mathbf{e}\right)$ and $\varphi_{\boldsymbol{\gamma}}=\arg (\boldsymbol{\gamma} \cdot \mathbf{e})$. The approximate results (71) and (72) are in reasonable agreement with the numerical solutions of Eqs. (68) and (70) beginning from the third pair of points $\left(\tau_{s}, x_{s}\right)$ (for the example presented in Fig. 3, the relative error for $\tau_{3}$ and $x_{3}$ is less than 3\% and $1 \%$, respectively, while for $\tau_{4}$ and $x_{4}$ the error is less than $2 \%$ and $0.6 \%$ ).

Finally, we note that the solutions $\left(\tau_{s}, x_{s}\right)$ with even $s$ do not contribute to the high-energy region near the rescattering plateau cutoff, while they are important for the low-energy part of the rescattering plateau. The boundary energy, $\bar{E}_{n}$, between these two regions of the LAES spectrum is governed by the parameter $\bar{\gamma}_{n}$, which is the limiting value of $\gamma_{n}^{(s)}$ as $s \rightarrow \infty$, where $\gamma_{n}^{(2 k-1)}$ for odd $s$ approaches $\bar{\gamma}_{n}$ from above, while $\gamma_{n}^{(2 k)}$ for even $s$ approaches it from below. The equation for $\bar{\gamma}_{n}$ follows from Eq. (69): $\bar{\gamma}_{n}^{2}=2\left|\bar{\gamma}_{n} \cdot \mathbf{e}\right|$. Using the parametrization (5) for the scalar product $\left(\bar{\gamma}_{n} \cdot \mathbf{e}\right)$, the boundary energy $\bar{E}_{n}=2 u_{p} \bar{\gamma}_{n}^{2}$ can be expressed as follows:

$$
\bar{E}_{n}=4 u_{p} \sin \theta_{\mathbf{p}_{n}}\left(1+\ell \cos 2 \phi_{\mathbf{p}_{n}}\right),
$$

where $\theta_{\mathbf{p}_{n}}$ and $\phi_{\mathbf{p}_{n}}$ are the polar and azimuthal angles for the vector $\mathbf{p}_{n}\left(\right.$ or $\left.\boldsymbol{\gamma}_{n}\right)$ in the basis $(\hat{\boldsymbol{\epsilon}},[\hat{\boldsymbol{\kappa}} \times \hat{\boldsymbol{\epsilon}}], \hat{\boldsymbol{\kappa}})$.

\section{B. Analytic formulas for the scattering amplitude}

Due to the coalescence of the two saddle points $\tau_{ \pm}^{(s)}$ for each $s$, the ordinary saddle-point method must be modified in order to evaluate analytically the integral in Eq. (65) (which determines the LAES amplitude within the rescattering approximation). For this purpose we use the modification suggested in Ref. [39] and used recently to obtain factorized results for HHG [23] and ATI [25] yields. This modification consists in approximating the phase factor $\phi_{s}(\tau)$ by a cubic polynomial in the neighborhood of the point $\tau=\tau_{s}$, followed by removing from the integral (65) the slowly oscillating preexponential factor at $\tau=\tau_{s}$ and extending the range of integration to $\pm \infty$. The amplitude $\mathcal{A}_{n}^{(1)}$ can then be evaluated analytically in terms of an Airy function, $\operatorname{Ai}(x)$ [37]. The standard uniform approximation (in which one approximates the smooth preexponential factor by a linear function in the interval between the points $\tau=\tau_{ \pm}^{(s)}$ ) [35,36]) gives approximately the same accuracy of results, but leads to cumbersome formulas, which are less suitable for further analyses and physical interpretations.

As discussed above, the function $\phi_{s}(\tau)$ is approximated as follows:

$\phi_{s}(\tau) \approx \tilde{\phi}_{s}+\frac{\mathbf{P}_{n}^{2}\left(\tau_{s}\right)-\mathbf{Q}^{2}\left(\tau_{s}, \tau_{s}^{\prime}\right)}{2 m_{e} \hbar \omega}\left(\tau-\tau_{s}\right)+\frac{\alpha_{s} u_{p}}{3 \hbar \omega}\left(\tau-\tau_{s}\right)^{3}$,

where $\tau_{s}^{\prime}=\tau_{s}-x_{s}, \tilde{\phi}_{s} \equiv \phi_{s}\left(\tau_{s}\right)$, and the dimensionless factor $\alpha_{s}$ is proportional to the third derivative of $\phi_{s}(\tau)$ at $\tau=\tau_{s}$, where in calculating this derivative one must take into account the $\tau$ dependence of $x_{s}(\tau)$, defined implicitly by Eq. (68). One obtains

$$
\alpha_{s}=2\left(\boldsymbol{v}_{s}-\boldsymbol{\gamma}_{n}\right) \cdot \operatorname{Im}\left(\mathbf{e} e^{-i \tau_{s}}\right)+\Delta \alpha_{s},
$$

where $\boldsymbol{v}_{s} \equiv \boldsymbol{v}\left(\tau_{s}, \tau_{s}^{\prime}\right)$ and

$$
\Delta \alpha_{s}=\left.\frac{d^{3}}{d \tau^{3}}\left\{\left[x_{s}(\tau)-x_{s}\right]\left(\boldsymbol{v}-\boldsymbol{v}_{s}\right)^{2}\right\}\right|_{\tau=\tau_{s}} .
$$

The explicit form of $\Delta \alpha_{s}$ is cumbersome. It is not presented here because numerical evaluation shows that it gives only a minor contribution to the final results.

Evaluating now the integral (65), we take into account that the amplitudes $\mathcal{A}\left[\mathbf{P}\left(\tau_{s}^{\prime}\right), \mathbf{Q}\left(\tau_{s}^{\prime}, \tau\right)\right]$ and $\mathcal{A}\left[\mathbf{Q}\left(\tau, \tau_{s}^{\prime}\right), \mathbf{P}_{n}(\tau)\right]$ depend only weakly on $\tau$ in the neighborhood of the saddle points $\tau_{ \pm}^{(s)}$ [which satisfy Eqs. (46) and (67)]. Thus, the amplitude $\mathcal{A}$, evaluated at $\tau=\tau_{s}$, can be replaced by the (on shell) amplitude $\mathcal{A}_{\mathrm{el}}$ of field-free elastic electron scattering. The result for the LAES amplitude $\mathcal{A}_{n}^{(1)}$ is

$$
\mathcal{A}_{n}^{(1)}=\frac{1}{\alpha_{0}} \sum_{s=1}^{\infty} D_{s} \mathcal{A}_{\mathrm{el}}\left(\mathbf{P}^{(s)}, \mathbf{Q}^{(s)}\right) \mathcal{A}_{\mathrm{el}}\left(\mathbf{Q}^{(s)}, \mathbf{P}_{n}^{(s)}\right),
$$


where $\quad \mathbf{P}^{(s)} \equiv \mathbf{P}\left(\tau_{s}^{\prime}\right), \quad \mathbf{P}_{n}^{(s)} \equiv \mathbf{P}_{n}\left(\tau_{s}\right), \quad \mathbf{Q}^{(s)} \equiv \mathbf{Q}\left(\tau_{s}^{\prime}, \tau_{s}\right), \quad$ and $\mathbf{Q}^{(s)} \equiv \mathbf{Q}\left(\tau_{s}, \tau_{s}^{\prime}\right)$. The factors $D_{s}$ in Eq. (76) are expressed in terms of the Airy function:

$$
D_{s}=\left(\frac{\hbar \omega}{u_{p}}\right)^{1 / 3} \frac{e^{i \tilde{\phi}_{s}} \operatorname{Ai}\left(\zeta_{s}\right)}{\alpha_{s}^{1 / 3} \sqrt{x_{s}^{3} \beta_{s}}},
$$

where $\beta_{s} \equiv \beta\left(\tau_{s}\right)$ is given by Eq. (43), and

$$
\zeta_{s}=\frac{\left[\left(\mathbf{P}_{n}^{(s)}\right)^{2}-\left(\mathbf{Q}^{(s)}\right)^{2}\right] /(2 m)}{u_{p}\left[\alpha_{s}\left(\hbar \omega / u_{p}\right)^{2}\right]^{1 / 3}} .
$$

The expression (76) may be simplified after further analysis and some additional approximations. First, in accordance with the above analysis of the solutions of the saddle-point equations, the sum over $s$ in Eq. (76) can be split into separate sums over odd $s$ and even $s$. The sum over even $s$ contributes to the scattering amplitude only in the low-energy part of the rescattering plateau defined by $E_{n}<\bar{E}_{n}$ [cf. Eq. (73)]. Second, the contribution of each succeeding term of the sum in Eq. (76) decreases because the coefficient $D_{s}$ decreases as $D_{s} \sim x_{s}^{-3 / 2}$. Furthermore, each succeeding odd $(s=2 k+1)$ term contributes negligibly to the scattering amplitude in the region $\gamma_{n}>\gamma_{n}^{(s)}$ because the Airy function $\operatorname{Ai}\left(\zeta_{s}\right)$ decreases exponentially for $\zeta_{s}>-1.019$. Thus, we assume that the term with $s=1$ gives the dominant contribution in the region of the rescattering plateau cutoff, that the term with $s=2$ contributes most to the region of the onset of the plateau, and that other terms (with higher $s$ ) give corrections in the intermediate region. Finally, the amplitude for field-free elastic scattering is a smooth function of its arguments and changes only slightly with respect to variations of $s$ having the same parity, owing to the quasiequidistant feature of the solutions $\left(\tau_{s}, x_{s}\right)$ [cf. Eqs. (71) and (72)]. These considerations allow us to approximate the amplitude $\mathcal{A}_{n}^{(1)}$ by separating the summation over $s$ in Eq. (76) into two sums (over odd and even $s$ ) and by removing the slowly varying amplitudes $\mathcal{A}_{\mathrm{el}}$, evaluated at the proper momenta, from under each summation. Since the main contributions to the sum (76) are given by the first terms of the two separate summations (for odd and even $s$ ), we assume that the momenta are the corresponding instantaneous kinetic momenta, evaluated at the (dimensionless) times $\left(\tau_{1}, \tau_{1}^{\prime}\right)$ for the odd $s$ sum (that is, $\mathbf{P}=\mathbf{P}^{(1)}, \mathbf{P}_{n}=\mathbf{P}_{n}^{(1)}, \mathbf{Q}^{\prime}=\mathbf{Q}^{\prime(1)}, \mathbf{Q}=\mathbf{Q}^{(1)}$ ), and evaluated at the times $\left(\tau_{2}, \tau_{2}^{\prime}\right)$ for the even $s$ sum (that is, $\left.\tilde{\mathbf{P}}=\mathbf{P}^{(2)}, \tilde{\mathbf{P}}_{n}=\mathbf{P}_{n}^{(2)}, \tilde{\mathbf{Q}}^{\prime}=\mathbf{Q}^{\prime(2)}, \tilde{\mathbf{Q}}=\mathbf{Q}^{(2)}\right)$. The result is

$$
\begin{aligned}
\mathcal{A}_{n}^{(1)}\left(\mathbf{p}, \mathbf{p}_{n}\right)= & \frac{1}{\alpha_{0}}\left[D^{(o)} \mathcal{A}_{\mathrm{el}}\left(\mathbf{P}, \mathbf{Q}^{\prime}\right) \mathcal{A}_{\mathrm{el}}\left(\mathbf{Q}, \mathbf{P}_{n}\right)\right. \\
& \left.+D^{(e)} \mathcal{A}_{\mathrm{el}}\left(\tilde{\mathbf{P}}, \tilde{\mathbf{Q}}^{\prime}\right) \mathcal{A}_{\mathrm{el}}\left(\tilde{\mathbf{Q}}, \tilde{\mathbf{P}}_{n}\right)\right],
\end{aligned}
$$

where $D^{(o)}=\sum_{k=0}^{\infty} D_{2 k+1}, D^{(e)}=\sum_{k=1}^{\infty} D_{2 k}$.

The approximate result (79) [as well as the more accurate result (76)] shows that the LAES amplitude with account of rescattering effects is given by a sum of factorized terms: All effects of the scattering potential $U(r)$ are collected in the two exact amplitudes $\mathcal{A}_{\mathrm{el}}$ for field-free elastic electron scattering, while the factors $D_{s}$ [defined by Eq. (77) in terms of an Airy function] depend only on the laser parameters. Therefore, neither the scattering amplitude nor the LAES cross section can be factorized over the entire rescattering plateau region as a product of only two (laser and atomic) factors; however, such a factorization becomes possible in the highenergy part of the rescattering plateau, due to the negligible contribution of the second term in Eq. (79) in this region.

\section{FACTORIZATION OF THE LAES CROSS SECTION IN THE RESCATTERING PLATEAU REGION}

\section{A. Three-step formula for the LAES cross section}

In the high-energy part of LAES spectrum, we can neglect the second term of Eq. (79) for the LAES amplitude in the rescattering approximation as well as the first (Kroll-Watson) term in Eq. (64). Substituting Eq. (79) into Eq. (27), we obtain a factorized result for the LAES differential cross section in the high-energy region of the rescattering plateau:

$$
\frac{d \sigma_{n}^{(r)}\left(\mathbf{p}, \mathbf{p}_{n}\right)}{d \Omega_{\mathbf{p}_{n}}}=\frac{d \sigma_{\mathrm{el}}\left(\mathbf{P}, \mathbf{Q}^{\prime}\right)}{d \Omega_{\mathbf{Q}^{\prime}}} \mathcal{W}\left(\mathbf{p}, \mathbf{p}_{n}\right) \frac{d \sigma_{\mathrm{el}}\left(\mathbf{Q}, \mathbf{P}_{n}\right)}{d \Omega_{\mathbf{P}_{n}}},
$$

where the factor $\mathcal{W}\left(\mathbf{p}, \mathbf{p}_{n}\right)$,

$$
\mathcal{W}\left(\mathbf{p}, \mathbf{p}_{n}\right)=\frac{p_{n}}{\alpha_{0}^{2} p}\left|\sum_{k=0}^{\infty} D_{2 k+1}\right|^{2},
$$

depends on the momenta $\mathbf{p}$ and $\mathbf{p}_{n}$ of the incident and scattered electrons through the explicit dependence of the instantaneous momentum $\mathbf{P}_{n}^{(s)}\left[=\mathbf{p}_{n}-e \mathbf{A}\left(\tau_{s}^{\prime}\right) / c\right]$ in the argument of the Airy function in Eq. (77) and through the implicit dependence of the times $\tau_{s}=\tau_{s}\left(\mathbf{p}, \mathbf{p}_{n}\right)$ and $\tau_{s}^{\prime}=\tau_{s}^{\prime}\left(\mathbf{p}, \mathbf{p}_{n}\right)$ on the momenta $\mathbf{p}$ and $\mathbf{p}_{n}$. Since Eq. (80) was obtained as a simplified, low-frequency version of the exact quantum results for the scattering problem, its expression in terms of three factors provides a convincing quantum justification of the classical three-step rescattering scenario of the LAES process for the general case of an elliptically polarized laser field.

The cross section $d \sigma_{\mathrm{el}}\left(\mathbf{P}, \mathbf{Q}^{\prime}\right) / d \Omega_{\mathbf{Q}^{\prime}}$ in Eq. (80) describes the elastic scattering of an electron with initial momentum p from the potential $U(r)$ at the time moment $t^{\prime}=\tau_{1}^{\prime} / \omega$. Since the collision takes place in the presence of a field $\mathbf{F}(t)$, this term involves (instead of the momentum p) the laser-modified instantaneous momentum $\mathbf{P}$ of the electron at the moment of collision. The scattering direction is given by the vector $\mathbf{Q}^{\prime}$, which is determined only by the vector potential of the elliptically polarized laser field and has the sense of an intermediate "kinetic momentum" of the electron in an "intermediate" state, immediately after the elastic scattering event at the moment $t^{\prime}$. From this state the electron starts to move in the laser field up to the moment of the second scattering (or rescattering). The cross section $d \sigma_{\mathrm{el}}\left(\mathbf{P}, \mathbf{Q}^{\prime}\right) / d \Omega_{\mathbf{Q}^{\prime}}$, involving the instantaneous momenta $\mathbf{P}$ and $\mathbf{Q}^{\prime}$, describes elastic scattering (since $\left|\mathbf{Q}^{\prime}\right|=|\mathbf{P}|$ ), while the initial momentum $\mathbf{p}$ changes to $\mathbf{q}(|\mathbf{p}| \neq|\mathbf{q}|)$. In order to ensure the condition for return of the electron back to the origin [where the magnitude of the potential $U(r)$ is maximal] at the moment $t$, the vector $\mathbf{q}=\mathbf{q}\left(\tau_{1}, \tau_{1}^{\prime}\right)$ depends on two times: the time $t^{\prime}$ of the first collision and the time $t=\tau_{1} / \omega$ of rescattering. The result of the rescattering at the moment $t$ is that the electron with the intermediate momentum $\mathbf{q}$ rescatters along the direction of the final (detected) momentum $\mathbf{p}_{n}$. This event is described in Eq. (80) by the cross section $d \sigma_{\mathrm{el}}\left(\mathbf{Q}, \mathbf{P}_{n}\right) / d \Omega_{\mathbf{P}_{n}}$ for field-free elastic scattering with instantaneous momenta $\mathbf{Q}$ and $\mathbf{P}_{n}$ (where $\left|\mathbf{P}_{n}\right|=|\mathbf{Q}|$ ). 
The key factor in the factorized cross section (80) is the propagation factor $\mathcal{W}\left(\mathbf{p}, \mathbf{p}_{n}\right)$. This factor describes the motion of a free electron in the field $\mathbf{F}(t)$ for the time $\Delta t=t-t^{\prime}$, resulting in the change of its initial kinetic momentum $\mathbf{P}$ to $\mathbf{P}_{n}$. Indeed, as is seen from the explicit form for $D_{s=2 k+1}$ in Eq. (77), the expression (81) for $\mathcal{W}\left(\mathbf{p}, \mathbf{p}_{n}\right)$ does not involve any dependence on the potential $U(r)$ and is determined completely by the free electron motion in the field $\mathbf{F}(t)$. Our numerical analysis shows that the sum over $k$ in Eq. (81) converges rapidly for arbitrary electron energy $E_{n}$ in the rescattering plateau region, so that only the first few terms in this sum over the saddle points contribute significantly. These terms effectively take into account both short and long closed trajectories of the electron in the laser field. These trajectories correspond to the two solutions, $\tau_{ \pm}^{(s)}$, of the saddle-point equations (68) and (69) whose interference causes the oscillatory features in the LAES spectra, which originate mathematically from the behavior of the Airy function $\operatorname{Ai}\left(\zeta_{s}\right)$. The times $t_{s}=\tau_{s} / \omega$ and $\Delta t_{s}=x_{s} / \omega$, which govern the magnitude of $D_{s}$ in Eq. (77), are, respectively, the moment of rescattering and the excursion time for electron propagation along the closed trajectory corresponding to the extreme path for which the $s$ th pair of short and long trajectories coalesce [as shown in Fig. 3(b)]. The numerator of the Airy function argument $\zeta_{s}$ in Eq. (78) represents the difference between the kinetic energy of the electron with final momentum $\mathbf{p}_{n}$ and the maximum classical energy, $\left(\mathbf{Q}^{(s)}\right)^{2} /(2 m)$, that can be gained by an electron with initial momentum $\mathbf{p}$ in the laser field before the rescattering event.

The physical interpretation of Eq. (80) is most clear if we limit ourselves to the case of the high-energy plateau cutoff region in the LAES spectrum, for which only the first term of the sum in Eq. (81) dominates and the factor $\mathcal{W}$ involves only a single term, $D_{1}$ :

$$
\mathcal{W}\left(\mathbf{p}, \mathbf{p}_{n}\right) \approx \frac{p_{n}}{\alpha_{0}^{2} p}\left|D_{1}\right|^{2}
$$

For the case of linear polarization $(\ell=1)$, the factorization (80) with $\mathcal{W}\left(\mathbf{p}, \mathbf{p}_{n}\right)$ given by Eq. (82) coincides with that obtained in Ref. [13]. The result (82) takes into account only the return of the electron along the first pair of short and long closed classical trajectories in Fig. 3(b), while the terms with $k \geqslant 1$ in the sum over $k$ in Eq. (81) determine the correction to the propagation factor in Eq. (82) due to electron returns along other "odd" (with $s=2 k+1$ ) pairs of short and long trajectories [cf. Fig. 3(b)].

\section{B. Comparison with the exact TDER results}

In Figs. 4 and 5 we compare exact TDER results for $s$-wave scattering (cf. Sec. 1 of Appendix A) with the low-frequency analytic results (for effective range theory parameters $a_{0}$ and $r_{0}$ corresponding to the case of $e-\mathrm{H}$ scattering). One sees that the analytic result (76) for the scattering amplitude describes well the entire rescattering plateau region of the LAES spectra [we find that the simpler two-term result (79) for $\mathcal{A}_{n}^{(1)}\left(\mathbf{p}, \mathbf{p}_{n}\right)$ provides the same accuracy in describing the rescattering plateau]. For the high-energy part of the plateau $\left(E_{n}>\bar{E}_{n}\right)$, the three-step formula (80) is in good agreement with the exact results. Moreover, the main contribution is given by the term

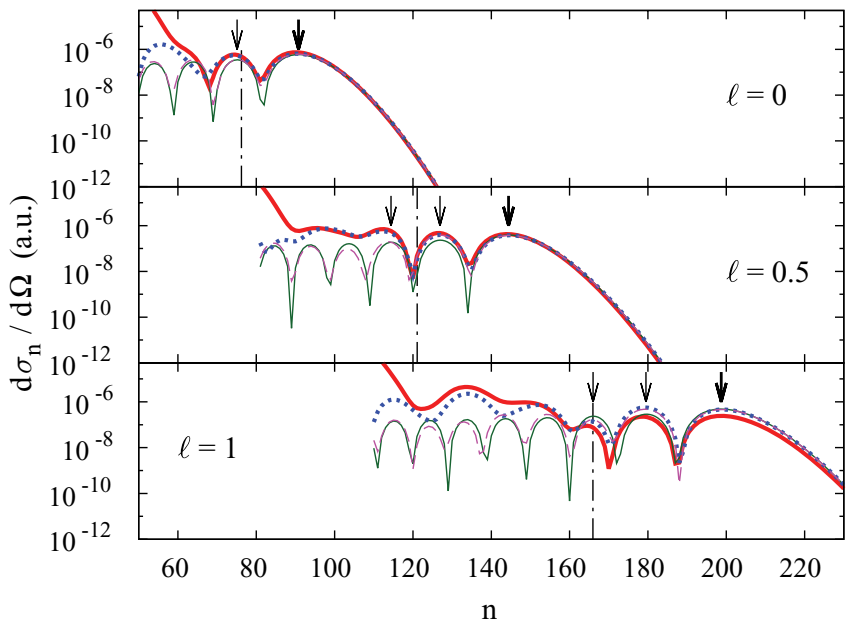

FIG. 4. (Color online) LAES differential cross section for forward $s$-wave $e$-H scattering $\left(\mathbf{p}\left\|\mathbf{p}_{n}\right\| \hat{\boldsymbol{\epsilon}}\right)$ as a function of the number $n$ of absorbed laser photons in an elliptically polarized $\mathrm{CO}_{2}$-laser field with $\hbar \omega=0.117 \mathrm{eV}(\lambda=10.6 \mu \mathrm{m})$ and intensity $I=2.5 \times 10^{11} \mathrm{~W} / \mathrm{cm}^{2}$ for three different degrees of linear polarization $\ell(=0,0.5,1)$ and incident electron energy $E=1.58 \mathrm{eV}$. Thick solid lines, exact TDER results; dotted lines, result (76) for the LAES amplitude; dashed lines, the three-step formula (80); thin solid lines, Eq. (80) with approximation (82) for the propagation factor. Vertical dotted-dashed lines mark the position of the boundary [cf. Eq. (73)] between the two regions of the rescattering plateau. Arrows indicate the positions of the interference maxima and the plateau cutoffs.

corresponding to the shortest excursion time of the electron along the closed trajectory [cf. Eq. (82)]. The account of the longer trajectories [given by the terms in Eq. (81) with $k>0$ ] provides a correction to the result (82) in the spectral region between $\bar{E}_{n}$ and the energy corresponding to the last (closest to the plateau cutoff) oscillatory minimum.

Our analysis shows that the agreement between the analytic formula and the exact results in the cutoff region worsens for $\ell \rightarrow 1$ (cf. Fig. 4). This fact is connected with the loss of the contributions to the scattering amplitude of the intermediate QES-channels with negative quasienergies $\epsilon_{n}=E+u_{p}+$ $n \hbar \omega$ [cf. Eq. (A7)] when the saddle-point approximation for the exact TDER equations was made. The effect of the closed channels on the LAES amplitude is not considered in this paper. We just note that the contributions of the closed channels to the LAES cross section in the high-energy plateau region noticeably depends on the laser intensity and the incident electron energy $E$ for a linearly polarized field and disappears for the case of circular polarization.

The comparison of our analytic results with exact TDER results for $p$-wave scattering (cf. Sec. 2 of Appendix A) is presented in Figs. 6 and 7, where the effective range theory parameters are those for $e$-F scattering: $\left|E_{0}\right|=3.401$ $\mathrm{eV}, \kappa=0.500$ a.u., $a_{1}=0.827 \kappa^{-3}$, and $r_{1}=-4.417 \kappa$. The intensity, $I=6.92 \times 10^{12} \mathrm{~W} / \mathrm{cm}^{2}$, of a midinfrared laser field with $\hbar \omega=0.354 \mathrm{eV}(\lambda=3.5 \mu \mathrm{m})$ and the incident electron energy, $E=4.78 \mathrm{eV}$, are chosen so that the ratios $u_{p} /(\hbar \omega)$ and $E /(\hbar \omega)$ have the same values as for $s$-wave scattering in Figs. 4 and 5. One sees that the accuracy of the analytic result (76) for the scattering amplitude and of the three-step formula (80) for 



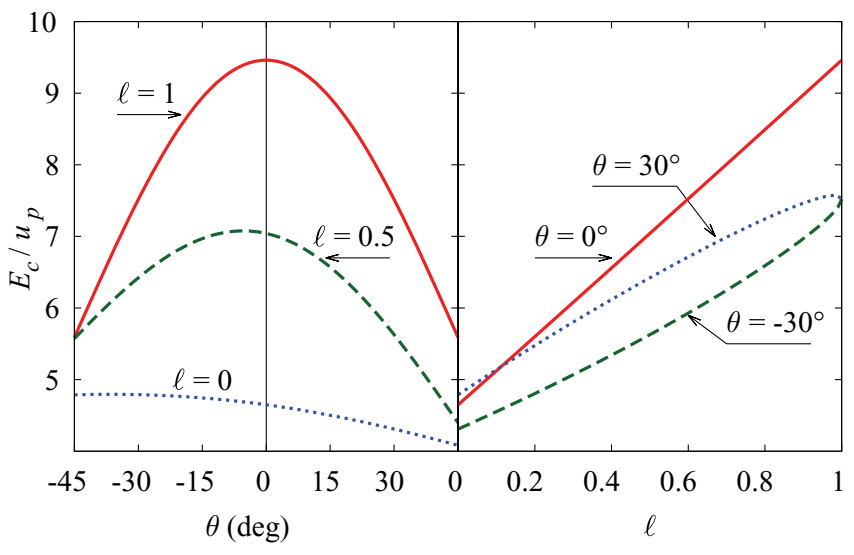

FIG. 8. (Color online) The cutoff energy $E_{c}$ vs scattering angle $\theta$ for different values of the linear polarization degree $\ell$ (left) and $E_{c}$ vs $\ell$ for different $\theta$ (right). The scattering geometry is $\mathbf{p} \| \hat{\boldsymbol{\epsilon}}, \mathbf{p}_{n} \perp \hat{\boldsymbol{\kappa}}$ and the laser parameters and the incident electron energy are the same as in Fig. 4.

smoother with decreasing linear polarization degree $\ell$. For forward scattering along the direction of the major axis of the polarization ellipse, the dependence of $E_{c}(\ell)$ on $\ell$ is close to linear over a wide interval of incident electron energies $E$ and laser intensities $I\left[I=c F^{2} /(8 \pi)\right]: E_{c}(\ell) / u_{p} \approx a_{1}(E, F)+$ $a_{2}(E, F) \ell$, where $a_{1,2}(E, F)$ are smooth functions of $E$ and $F$ (cf. Fig. 8).

Another noticeable effect seen in Fig. 8 is an asymmetry in the cutoff position with respect to the sign of the angle $\theta$ for $\ell<1$ (cf. also Figs. 5 and 7). (For the geometry $\mathbf{p} \| \hat{\boldsymbol{\epsilon}}, \mathbf{p}_{n} \perp \hat{\boldsymbol{\kappa}}$, one has $p_{n} \cos \theta=\mathbf{p}_{n} \cdot \hat{\boldsymbol{\epsilon}}$ and $p_{n} \sin \theta=\mathbf{p}_{n} \cdot[\hat{\boldsymbol{\kappa}} \times \hat{\boldsymbol{\epsilon}}]$, so that the positive direction of $\theta$ coincides with the direction of the field rotation for $\eta>0$.) This dichroic effect for the cutoff of the rescattering plateau in LAES spectra was predicted in Ref. [11].

The oscillation pattern in the dependence of $\mathcal{W}\left(\mathbf{p}, \mathbf{p}_{n}\right)$ on $\mathbf{p}_{n}$ originates from the interference of two classical electron trajectories, which merge at the cutoff with the shortest extremal trajectory and which were taken into account in evaluating the LAES amplitude (cf. discussion in Sec. V A). This interference explains the oscillatory patterns in the LAES spectra below the plateau cutoff (for $\zeta_{1}<z_{1}$ ), which are known from numerical calculations (cf. Ref. [11] and Figs. 4-7) and were discussed in Refs. [13] and [40]. [In Ref. [40] the origin of the oscillatory patterns as a consequence of the interference between real electron trajectories was established by taking into account the scattering potential $U(r)$ perturbatively within the strong-field and uniform approximations.]

The positions of the minima and maxima of the interference oscillations may be found in the same way as for the cutoff position, that is, by solving the system (68) and (70) and Eq. (83) for $\boldsymbol{\gamma}_{n}=\boldsymbol{\gamma}_{n, \min / \max }$, replacing $z_{1}$ with $z_{k}$ [where $z_{k}$ are the positions of the zeros and the maxima of $\left.\operatorname{Ai}^{2}\left(\zeta_{1}\right)\right]$. For $k \geqslant 2$, the values of $z_{k}$ are well approximated by equating to $\pi k / 2$ the argument of the sine function in the asymptotic form of $\mathrm{Ai}\left(-\left|\zeta_{1}\right|\right)$ for large $\left|\zeta_{1}\right|[37]$,

$$
\operatorname{Ai}\left(-\left|\zeta_{1}\right|\right) \propto\left|\zeta_{1}\right|^{-1 / 4} \sin \left(\frac{2}{3}\left|\zeta_{1}\right|^{3 / 2}+\frac{\pi}{4}\right) .
$$

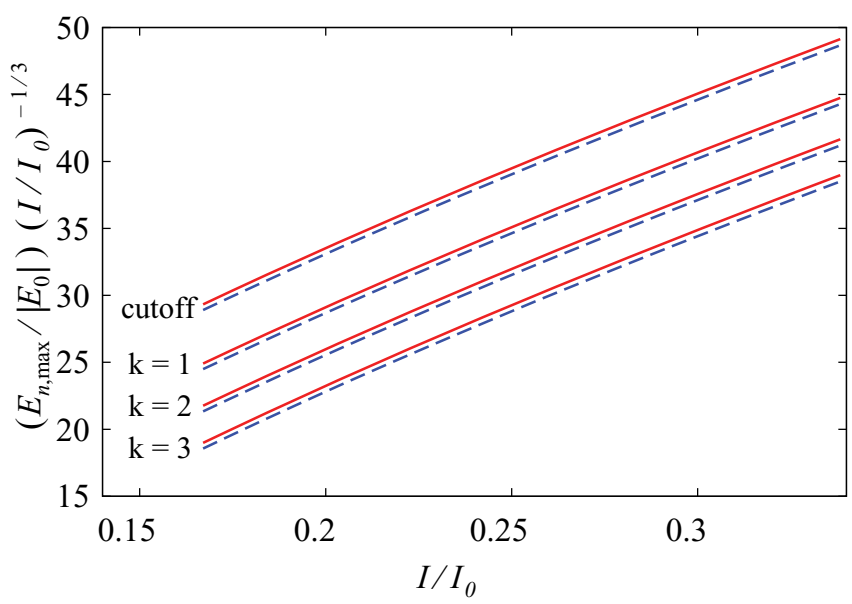

FIG. 9. (Color online) Scaled positions $\left(E_{n, \max }\right)$ of the plateau cutoff and of the oscillatory maxima closest to the cutoff (marked by $k=1,2,3)$ vs scaled laser intensity $I / I_{0}$ for forward scattering in a circularly polarized field and for two electron energies: $E=$ $13.5 \hbar \omega$ (solid lines) and $E=19.5 \hbar \omega$ (dashed lines). [Scaled units of intensity, $I_{0}=1.5 \times 10^{12} \mathrm{~W} / \mathrm{cm}^{2}$, and of energy, $\left|E_{0}\right|=0.755 \mathrm{eV}$, correspond to $e-\mathrm{H}$ scattering (see text). For the case of a $\mathrm{CO}_{2}$ laser: $E=1.58$ and $2.28 \mathrm{eV}$.]

The maxima and zeros of $\operatorname{Ai}^{2}\left(\zeta_{1}\right)$ (and hence the maxima and minima of $d \sigma_{n} / d \Omega$ ) correspond to odd and even $k$ respectively in the relation

$$
\zeta_{1}=z_{k}=-0.25[2 \pi(2 k-1)]^{2 / 3}, \quad k \geqslant 2 .
$$

The estimated positions of a few maxima in the LAES spectra closest to the cutoff are indicated in Figs. 4-7 by arrows. One sees that these positions coincide well with the positions of the maxima in the exact TDER results.

We have found that the positions of the maxima or minima in the oscillatory LAES spectra depend on the scattering angle and on the laser polarization in much the same way as shown for the cutoff position, $E_{c}(\theta, \ell)$, in Fig. 8. However, the distance between the positions of the maxima or minima for fixed $\ell$ and $\theta$ depends essentially only on the laser intensity and scales as $I^{1 / 3}$. This fact is shown in Fig. 9 for the case of forward $e-\mathrm{H}$ scattering in a circularly polarized $(\ell=0)$ field for two values of the electron energy $E$. [The scaled unit of intensity, $I_{0}$, in Fig. 9 is defined as $I_{0}=c F_{0}^{2} /(8 \pi)$, where $F_{0}=$ $\sqrt{8 m\left|E_{0}\right|^{3}} /(|e| \hbar)$. Thus, for $e-\mathrm{H}$ scattering $\left(\left|E_{0}\right|=0.755 \mathrm{eV}\right)$, $I_{0}=1.5 \times 10^{12} \mathrm{~W} / \mathrm{cm}^{2}$.] Note that for a linearly polarized field, the same intensity dependence for the positions of the maxima and minima was found analytically for LAES [13] and for ATD [25] processes.

Because of the sensitivity of the oscillatory patterns in the LAES spectra to the scattering angle (cf. Figs. 5 and 7), the angle-integrated spectra are smooth, as shown in the bottom panels in Figs. 5 and 7, in which the integration was performed over the "forward scattering" hemisphere: $0 \leqslant \theta_{\mathbf{p}_{n}} \leqslant 180^{\circ}$, $-90^{\circ} \leqslant \phi_{\mathbf{p}_{n}} \leqslant 90^{\circ}$, where $\theta_{\mathbf{p}_{n}}$ and $\phi_{\mathbf{p}_{n}}$ are the polar and azimuthal angles for the vector $\mathbf{p}_{n}$. For this case, one sees in Figs. 5 and 7 that the simple analytic result (80) with propagation factor (82) provides good agreement with the exact TDER results over the entire rescattering plateau. 


\section{CONCLUSIONS AND PERSPECTIVES}

Nowadays the manifestation of field-free atomic dynamics in strong-field processes and the retrieval of information on this dynamics from the measured outcomes of laser-atom interactions are attracting increasing interest. For HHG and ATI processes, this dynamical information can be obtained theoretically most convincingly using well-developed algorithms for direct numerical solution of the time-dependent Schrödinger equation. However, for laser-assisted collisions, numerical algorithms for calculating the scattering state wave function in an intense, low-frequency laser field have not yet been developed, even for the case of linear laser polarization. Moreover, the widely used strong-field approximation is not applicable for this purpose since for an electron in the continuum it treats the scattering potential perturbatively, using the Born approximation. Thus, for collision problems, nonperturbative approximate theories or exactly solvable models play an essential role in providing a deeper understanding of the influence of the scattering potential on laser-assisted collision processes.

In this paper, we have obtained quantum-mechanically (in the low-frequency limit) analytic expressions for cross sections of electron scattering from a potential in the presence of an elliptically polarized laser field using TDER theory, which permits one to obtain not only an exact numerical solution for the LAES problem but also simple analytic results for a number of limiting cases. Our analytic derivations are based on the analytic representation of the exact TDER scattering state $\Phi_{\mathbf{p}}(\mathbf{r}, t)$ in Eq. (13) as a sum of two terms: the "zero-order" term, which corresponds to the low-frequency, Kroll-Watson result for the scattering state [cf. Eq. (5.12) in Ref. [15]], and the "rescattering correction," which takes into account the strong laser field modifications of the electron interaction with the scattering potential $U(r)$ beyond the Kroll-Watson approximation. Since the Kroll-Watson term in the LAES cross section decreases exponentially beyond the classically allowed region (for high $n$ ), the rescattering correction becomes dominant there and describes perfectly the rescattering plateau in the high-energy region of the LAES spectrum. The high accuracy of our analytic approximations for the exact TDER LAES amplitude is demonstrated by comparison of analytic and exact numerical TDER results for the ellipticity and angular dependencies of LAES spectra for two different cases: $s$-wave scattering (corresponding to electron scattering from hydrogen or an alkali-metal atom; cf. Figs. 4 and 5 for $e$-H scattering) and $p$-wave scattering (corresponding to a halogen atom target; cf. Figs. 6 and 7 for $e$-F scattering).

The key results of this paper are the expression (76) for the LAES amplitude in the rescattering approximation and the three-step formula (80) for the LAES cross section. The factorized result (80) describes well the high-energy part of the rescattering plateau, while the nonfactorized LAES amplitude (76) [as well as the two-term result (79)] describes the LAES spectrum over the entire rescattering plateau region (cf. Figs. 4-7). After substituting Eq. (82) for the propagation factor, the formula (80) provides a generalization of the result for a linearly polarized laser field [13] to the case of nonzero driving laser ellipticity.
The major limitation of the TDER theory model is that it takes into account only a single partial-wave scattering phase (in a given $l$-wave channel) for the potential $U(r)[41,42]$, whereas the entire set of phase shifts should be taken into account in describing elastic electron scattering by a neutral atom. However, this deficiency is compensated by the very clear and physically transparent interpretation of our key results (76) and (80). Indeed, (i) the quantum-mechanically derived factorized formula (80) agrees completely with the semiclassical three-step rescattering scenario for the LAES process, giving, in fact, a quantum "replica" (or quantum justification) of this scenario; (ii) the account of rescattering effects in our analysis was performed nonperturbatively in the potential $U(r)$, so that the results (76) and (80) contain the exact (non-Born) amplitude and cross section for elastic electron scattering by the potential $U(r)$ within the effective range theory; and (iii) the factors $D_{s}$ [cf. Eq. (77)] in Eq. (76), as well as the propagation factor $\mathcal{W}\left(\mathbf{p}, \mathbf{p}_{n}\right)$, do not involve any parameters of the potential $U(r)$ and thus are valid for any atomic target. [In particular, our results for the $s$-wave and the $p$-wave scattering show that these factors do not depend on the spatial symmetry of a bound state (if it exists) in an atomic potential $U(r)$.] Therefore, it is reasonable to expect that a generalization of Eqs. (76) and (80) beyond the TDER theory may be performed quite straightforwardly, specifically by replacing the field-free scattering amplitudes $\mathcal{A}_{\mathrm{el}}$ in Eq. (76) and the TDER cross sections $d \sigma_{\mathrm{el}} / d \Omega$ in Eq. (80) by the amplitudes and cross sections for elastic electron scattering by a particular real atom obtained from either experimental measurements or accurate theoretical calculations. Similar generalizations of factorized TDER results for HHG [24] and ATI [25] yields to the case of real atomic targets have been shown to provide fine agreement with results of accurate numerical solutions of the time-dependent Schrödinger equation for the plateau cutoff region in HHG and ATI spectra. For LAES, the aforementioned generalization allows one to extend the formulas (76) and (80) to the case of atomic targets (such as inert gases) which do not support a bound state of an attached electron (i.e., a negative ion) in spite of the fact that the description of LAES within the TDER theory presented in this paper is not applicable for such cases. The use of the results (76) and (80) for such cases that go beyond the present TDER theory will be described in a separate publication.

The results in this paper become inapplicable for resonant electron energies, $E \approx \mu \hbar \omega-\left|E_{0}\right|-u_{p}$, at which the electron may be temporarily captured in a bound state $\psi_{\kappa l m_{l}}(\mathbf{r})$ of the potential $U(r)$ by emitting $\mu$ photons [43], and for threshold energies, $E=k \hbar \omega, k=1,2, \ldots$, at which the LAES spectrum may be affected considerably by threshold phenomena, corresponding to the closing (or opening) of the channel for stimulated emission of $k$ laser photons by the incident electron [28]. Since both resonant and threshold phenomena have a purely quantum origin, in which case the discreteness of the photon energy $n \hbar \omega$ is essential, these phenomena disappear in the low-frequency approximation $(\hbar \omega \rightarrow 0)$ used in the present work. An analysis of resonant and threshold phenomena for the LAES process in an elliptically polarized laser field will be published elsewhere.

Finally, we note that even for the simplest geometry, $\mathbf{p} \| \hat{\boldsymbol{\epsilon}}$, the ellipticity $\eta$ of the laser field affects significantly the 
angular distribution (AD) of scattered electrons as compared to the case of linear polarization, because it destroys the axial symmetry of the AD that exists for $\eta=0$ with respect to the direction of $\hat{\boldsymbol{\epsilon}}$. In particular, the ADs for $\eta \neq 0$ differ substantially for $\eta= \pm|\eta|$, thus exhibiting an elliptic dichroism effect whose detailed study for both the low-energy and the rescattering regions of the LAES spectrum is now in progress.

\section{ACKNOWLEDGMENTS}

This work was supported in part by RFBR Grant No. 13-0200420, by NSF Grant No. PHY-1208059, and by the Russian Federation Ministry of Education and Science (Contract No. 14.B37.21.1937).

\section{APPENDIX A: THE MATRIX FORM OF THE TDER EQUATIONS FOR THE FOURIER COEFFICIENTS $f_{k}^{\left(l m_{l}\right)}(\mathbf{p})$ AND THE LAES AMPLITUDE}

\section{Results for $s$-wave scattering $(l=0)$}

Equation (23) can be converted into a system of inhomogeneous linear algebraic equations for the Fourier coefficients $f_{k}(\mathbf{p})$ of the function $f_{\mathbf{p}}(\tau)=\sum_{k} f_{k}(\mathbf{p}) e^{-i k \tau}$ :

$$
\sum_{s^{\prime}} \mathcal{M}_{s, s^{\prime}}(\epsilon+\delta \hbar \omega) f_{2 s^{\prime}+\delta}(\mathbf{p})=\kappa c_{2 s+\delta}(\mathbf{p}),
$$

where the symbol $\delta$ is equal to 0 (1) for an even (odd) $k$. The inhomogeneous term in the system (A1) is expressed in terms of Fourier coefficients of the wave function $\chi_{\mathbf{p}}(\mathbf{r}=0, \tau)$ [cf. Eq. (14)]:

$$
c_{k}(\mathbf{p})=i^{k} \mathcal{J}_{-k}^{*}\left(\frac{|e| F}{m \hbar \omega^{2}}(\mathbf{e} \cdot \mathbf{p}), \frac{\ell u_{p}}{2 \hbar \omega}\right),
$$

where $\mathcal{J}_{n}(z, x)$ is a generalized Bessel function,

$$
\mathcal{J}_{n}(z, x)=\sum_{p=-\infty}^{\infty} e^{i(n+2 p) \arg (z)} J_{n+2 p}(|z|) J_{p}(x) .
$$

Therefore, the system (A1) is equivalent to two separate (uncoupled) systems for even and odd Fourier coefficients of the QES wave function $\Phi_{\mathbf{p}}(\mathbf{r}, t)$ at $r \rightarrow 0$.

The matrix elements $\mathcal{M}_{s, s^{\prime}}(\epsilon)$ in Eq. (A1) have the following form:

$$
\begin{gathered}
\mathcal{M}_{s, s^{\prime}}(\epsilon)=\mathcal{A}^{-1}\left(\tilde{p}_{2 s}\right) \delta_{s, s^{\prime}}-M_{s, s^{\prime}}(\epsilon), \\
\mathcal{A}\left(\tilde{p}_{2 s}\right)=\frac{1}{-a_{0}^{-1}+r_{0} k_{2 s}^{2} / 2-i k_{2 s}}, \quad k_{2 s}=\frac{\tilde{p}_{2 s}}{\hbar}, \\
M_{s, s^{\prime}}(\epsilon)=i^{s-s^{\prime}} \sqrt{\frac{m \omega}{2 \pi i \hbar}} \int_{0}^{\infty} \frac{d \tau}{\tau^{3 / 2}} e^{\left.i \epsilon_{s+s^{\prime}} \tau / \hbar \omega\right)} \\
\times\left[e^{-i \lambda(\tau)} J_{s-s^{\prime}}(\ell z(\tau))-\delta_{s, s^{\prime}}\right], \\
\lambda(\tau)=\frac{u_{p}}{\hbar \omega}\left(\tau-\frac{4}{\tau} \sin ^{2} \frac{\tau}{2}\right), \\
z(\tau)=\frac{u_{p}}{\hbar \omega}\left(\sin \tau-\frac{4}{\tau} \sin ^{2} \frac{\tau}{2}\right),
\end{gathered}
$$

where $J_{n}(x)$ is a Bessel function, and the following notations are used in Eqs. (A3)-(A5): $\epsilon_{n} \equiv \epsilon+n \hbar \omega=E+u_{p}+n \hbar \omega$, $\tilde{p}_{n}=\sqrt{2 m \epsilon_{n}}$. Note that only diagonal matrix elements $\mathcal{M}_{s, s^{\prime}}$ contain the information on atomic dynamics [i.e., the field-free elastic scattering amplitude $\mathcal{A}\left(\tilde{p}_{2 s}\right)$ for a "momentum" $\tilde{p}_{2 s}$, which is imaginary for closed channels, with $\epsilon_{2 s}<0$ ], while the nondiagonal elements $\left(s \neq s^{\prime}\right)$ depend only on the incident electron energy $E$ and the laser parameters.

In terms of the coefficients $f_{k}(\mathbf{p})$, the LAES amplitude (26) can be represented in an alternative form [28]:

$$
\mathcal{A}_{n}\left(\mathbf{p}, \mathbf{p}_{n}\right)=\kappa^{-1} \sum_{k=-\infty}^{\infty} f_{k}(\mathbf{p}) c_{k-n}^{*}\left(\mathbf{p}_{n}\right) .
$$

The low-frequency iterative solution of the integrodifferential equation (23), presented in Sec. III, corresponds to the iterative account of the integral terms $M_{s, s^{\prime}}$ in Eq. (A5) for solving the system (A1). In the lowest order in $M_{s, s^{\prime}}$, the solution of Eq. (A1) is

$f_{k}(\mathbf{p}) \approx \kappa \mathcal{A}\left(\tilde{p}_{k}\right)\left[c_{k}-\sum_{s^{\prime}} \mathcal{A}\left(\tilde{p}_{k+2 s^{\prime}}\right) M_{0, s^{\prime}}\left(\epsilon_{k}\right) c_{k+2 s^{\prime}}\right]$.

The first term in the approximation (A7) corresponds to the zero-order approximation (37) for the function $f_{\mathbf{p}}(\tau)$, while the second term describes the rescattering correction (47). We emphasize that the approximation (A7) is more accurate than the low-frequency expansion (39) because the LAES amplitude (A6) [as well as the sum over $s^{\prime}$ in Eq. (A7)] involves a summation over all intermediate channels, including closed channels. However, using the approximation (A7) we are not able to provide a closed-form analytic expression for the LAES amplitude. Finally, we note that all nondiagonal matrix elements $M_{s, s^{\prime}}$ (with $s \neq s^{\prime}$ ) are equal to zero for a circularly polarized $(\ell=0)$ field $\mathbf{F}(t)$. In this case the sum over $s^{\prime}$ in Eq. (A7) contains only the single term with $s^{\prime}=0$.

\section{Results for $p$-wave scattering $(l=1)$}

For $l=1$, matching the QES wave function (13) [with $\Phi_{\mathbf{p}}^{(\mathrm{sc})}(\mathbf{r}, t)$ given by Eq. (18)] to the small- $r$ boundary condition (11) results in a system of three (for $\mu=0, \pm 1$ ) coupled integro-differential equations for the functions $f_{\mathbf{p}}^{(1 \mu)}(\tau)=$ $\sum_{k} f_{k}^{(1 \mu)}(\mathbf{p}) e^{-i k \tau}$ [cf. Eq. (23) for the case $l=0$ ]. This system can be converted into the following three matrix equations for the Fourier coefficients $f_{k}^{(\mu)} \equiv f_{k}^{(1 \mu)}(\mathbf{p})$ :

$$
\begin{gathered}
\sum_{s^{\prime}} M_{s, s^{\prime}}^{(0)}\left(\epsilon_{\delta}\right) f_{2 s^{\prime}+\delta}^{(0)}=\kappa^{2} c_{2 s+\delta}^{(0)}, \\
\sum_{s^{\prime}}\left(\begin{array}{cc}
\bar{M}_{s, s^{\prime}}^{(-1)}\left(\epsilon_{\delta}\right) & \hat{M}_{s, s^{\prime}}^{(-1)}\left(\epsilon_{\delta}\right) \\
\hat{M}_{s, s^{\prime}}^{(1)}\left(\epsilon_{\delta}\right) & \bar{M}_{s, s^{\prime}}^{(1)}\left(\epsilon_{\delta}\right)
\end{array}\right)\left(\begin{array}{c}
f_{2 s^{\prime}+\delta}^{(-1)} \\
f_{2 s^{\prime}+\delta}^{(1)}
\end{array}\right)=\kappa^{2}\left(\begin{array}{c}
c_{2 s+\delta}^{(-1)} \\
c_{2 s+\delta}^{(1)}
\end{array}\right),
\end{gathered}
$$

where $\epsilon_{\delta}=\epsilon+\delta \hbar \omega$ and $\delta$ is equal to 0 (1) for an even (odd) $k$, similar to the result for $s$-wave scattering in Eq. (A1). The coefficients $c_{k}^{(\mu)}$ on the right-hand sides of Eqs. (A8) and (A9) can be expressed in terms of the coefficients $c_{k}(\mathbf{p})$, given by 
Eq. (A2):

$$
\begin{aligned}
c_{k}^{(\mu)}(\mathbf{p})= & \frac{p}{\hbar} \sqrt{4 \pi} Y_{1 \mu}^{*}(\hat{\mathbf{p}}) c_{k}(\mathbf{p})+i \mu \sqrt{3(1+\ell)} \frac{|e| F}{4 \hbar \omega} \\
& \times\left[\left(1+\frac{\mu \xi}{1+\ell}\right) c_{k-1}(\mathbf{p})-\left(1-\frac{\mu \xi}{1+\ell}\right) c_{k+1}(\mathbf{p})\right],
\end{aligned}
$$

where the spherical harmonic $Y_{1 \mu}(\hat{\mathbf{p}})$ is defined as in Ref. [44].

The matrix elements $M_{s, s^{\prime}}^{(0)}(\epsilon), \bar{M}_{s, s^{\prime}}^{(\mu)}(\epsilon)$, and $\hat{M}_{s, s^{\prime}}^{(\mu)}(\epsilon)$ $(\mu= \pm 1)$ in Eqs. (A8) and (A9) have the form (cf. Ref. [22])

$$
\begin{aligned}
M_{s, s^{\prime}}^{(0)}(\epsilon)= & \left(-\frac{1}{a_{1}}+\frac{r_{1} k_{2 s}^{2}}{2}-i k_{2 s}^{3}\right) \delta_{s, s^{\prime}} \\
+ & \mathcal{C} \int_{0}^{\infty} \frac{d \tau}{\tau^{5 / 2}} e^{i \epsilon_{s+s^{\prime}} \tau /(\hbar \omega)}\left[e^{-i \lambda(\tau)} J_{s-s^{\prime}}(\ell z(\tau))-\delta_{s, s^{\prime}}\right], \\
\bar{M}_{s, s^{\prime}}^{(\mu)}(\epsilon)= & M_{s, s^{\prime}}^{(0)}(\epsilon)+\mathcal{C} \int_{0}^{\infty} \frac{d \tau}{\tau^{3 / 2}} e^{\left.i \epsilon_{s+s^{\prime}} \tau / \hbar \hbar \omega\right)-i \lambda(\tau)} \\
& \times\left\{\left[i \rho_{1}(\tau)+\mu \xi z(\tau)\right] J_{s-s^{\prime}}(\ell z(\tau))\right. \\
& \left.-\ell \rho_{2}(\tau) J_{s-s^{\prime}}^{\prime}(\ell z(\tau))\right\}, \\
\hat{M}_{s, s^{\prime}}^{(\mu)}(\epsilon)= & \mathcal{C} \int_{0}^{\infty} \frac{d \tau}{\tau^{3 / 2}} e^{i \epsilon_{s+s^{\prime}} \tau /(\hbar \omega)-i \lambda(\tau)} \\
& \times\left\{-i \ell \rho_{1}(\tau) J_{s-s^{\prime}}(\ell z(\tau))+\rho_{2}(\tau)\left[J_{s-s^{\prime}}^{\prime}(\ell z(\tau))\right.\right. \\
& \left.\left.+\frac{\mu \xi\left(s-s^{\prime}\right)}{\ell z(\tau)} J_{s-s^{\prime}}(\ell z(\tau))\right]\right\},
\end{aligned}
$$

where $J_{n}^{\prime}(z)$ is the derivative of the Bessel function and the following notations are used:

$$
\begin{aligned}
\mathcal{C} & =\frac{3 i^{s-s^{\prime}+1}}{\sqrt{2 \pi i}}\left(\frac{m \omega}{\hbar}\right)^{3 / 2}, \\
\rho_{1}(\tau) & =\frac{u_{p}}{\hbar \omega}\left(\frac{4}{\tau^{2}} \sin ^{2} \frac{\tau}{2}-\frac{2}{\tau} \sin \tau+\cos \tau\right), \\
\rho_{2}(\tau) & =\frac{u_{p}}{\hbar \omega}\left(\frac{4}{\tau^{2}} \sin ^{2} \frac{\tau}{2}-\frac{2}{\tau} \sin \tau+1\right) .
\end{aligned}
$$

Once the Fourier coefficients $f_{k}^{(\mu)}(\mathbf{p})$ are known, the exact TDER result for the $p$-wave LAES amplitude is given by

$$
\mathcal{A}_{n}^{(l=1)}\left(\mathbf{p}, \mathbf{p}_{n}\right)=\kappa^{-2} \sum_{\mu=-1}^{1} \sum_{k=-\infty}^{\infty} f_{k}^{(\mu)}(\mathbf{p}) c_{k-n}^{(\mu) *}\left(\mathbf{p}_{n}\right) .
$$

\section{APPENDIX B: THE UNIFORM ASYMPTOTIC APPROXIMATION OF THE INTEGRAL (53)}

In this Appendix, we describe the approach for the uniform asymptotic expansion of the integral (53). We note first that after replacing the integration variable $\tau$ in Eq. (53) by $x=\tau-\pi / 2-\varphi_{\mathbf{t}}$, the amplitude $\mathcal{A}_{n}^{(0)}$ is expressed in terms of the integral $I_{n}(\rho)$ :

$$
\mathcal{A}_{n}^{(0)}=i^{n} e^{i n \varphi_{\mathrm{t}}} I_{n}(\rho), \quad I_{n}(\rho)=\frac{1}{2 \pi} \int_{-\pi}^{\pi} f(x) e^{i \varphi(\rho, x)} d x,
$$

where $f(x)=\mathcal{A}\left(x+\pi / 2+\varphi_{\mathrm{t}}\right)$ is a periodic function of $x$ and $\varphi(\rho, x)=n x-\rho \sin x$. Assuming $\rho \gg 1$ and $\rho \geqslant|n|$, the main contributions to the integral $I_{n}(\rho)$ are given by the neighborhoods of the saddle points $x=x_{ \pm}$, satisfying the equation $d \varphi(x) / d x=0$ :

$$
x_{ \pm}= \pm \alpha, \quad \cos \alpha=\frac{n}{\rho}, \quad 0 \leqslant \alpha \leqslant \pi .
$$

Since the points $x_{ \pm}$tend toward each other and coalesce at $\alpha=$ 0 , following the general idea of the uniform approximations of integrals [36], we rewrite the preexponential function $f(x)$, explicitly extracting the term that approximates $f(x)$ in the neighborhood of the two coalescing saddle points. Taking into account the periodicity of $f(x)$, we rewrite it in the following form:

$$
f(x)=a_{0}+a_{1} \sin x+(\cos x-\cos \alpha) g(x),
$$

where $a_{0}$ and $a_{1}$ are easily determined to be

$$
a_{0}=\frac{f\left(x_{+}\right)+f\left(x_{-}\right)}{2}, \quad a_{1}=\frac{f\left(x_{+}\right)-f\left(x_{-}\right)}{2 \sin \alpha},
$$

and where $g(x)$ is an analytic, smooth, periodic function of $x$. After substituting Eq. (B3) into Eq. (B1), the integration of the first two terms of the expression (B3) can be performed analytically. The result for $I_{n}(\rho)$ is

$$
I_{n}(\rho)=a_{0} J_{n}(\rho)+i a_{1} J_{n}^{\prime}(\rho)+\tilde{I}_{n}(\rho),
$$

where $J_{n}(\rho)$ and $J_{n}^{\prime}(\rho)$ are the Bessel function and its derivative, while $\tilde{I}_{n}(\rho)$ is the remainder integral:

$$
\tilde{I}_{n}(\rho)=\frac{1}{2 \pi} \int_{-\pi}^{\pi}(\cos x-\cos \alpha) g(x) e^{i \varphi(\rho, x)} d x .
$$

Integrating $\tilde{I}_{n}(\rho)$ by parts, we obtain

$$
\tilde{I}_{n}(\rho)=\frac{1}{2 \pi i \rho} \int_{-\pi}^{\pi} \frac{d g(x)}{d x} e^{i \varphi(\rho, x)} d x .
$$

Comparing Eq. (B6) with Eq. (B1), one sees that the remainder term $\tilde{I}_{n}(\rho)$ has the same form as the original integral (B1), but contains a small parameter $\rho^{-1}$. Representing the function $d g(x) / d x$ in Eq. (B6) by the form (B3) and applying the same integration procedure as for $I_{n}(\rho)$, we find the asymptotic expansion of the integral $I_{n}(\rho)$ for large values of the parameter $\rho$.

For the case of a Kroll-Watson-like approximation, we neglect the remainder term $\tilde{I}_{n}(\rho)$ in Eq. (B4), which gives immediately the result (55) for the scattering amplitude $\mathcal{A}_{n}^{(0)}$.

Also, we recall here another asymptotic approximation of the integral (B1), which was suggested in Ref. [34], where the integration interval in Eq. (B1) was divided into two parts $(-\pi \geqslant x \geqslant 0$ and $0 \geqslant x \geqslant \pi)$ followed by taking into account the saddle points $x_{ \pm}$independently (as noncoalescing saddle points). The result is that the integral $I_{n}(\rho)$ can be expressed in terms of the Anger function, $J_{n}(\rho)$ (which coincides with the Bessel function for integer $n$ ), and the Weber function, $E_{n}(\rho)[37]:$

$$
I_{n}(\rho)=a_{+} J_{n}(\rho)+i a_{-} E_{n}(\rho), \quad a_{ \pm}=\frac{f\left(x_{+}\right) \pm f\left(x_{-}\right)}{2} .
$$


[1] P. Saliéres et al., Science 292, 902 (2001).

[2] W. Becker, F. Grasbon, R. Kopold, D. B. Milošević, G. G. Paulus, and H. Walther, Adv. At. Mol. Opt. Phys. 48, 35 (2002).

[3] D. B. Milošević and F. Ehlotzky, Adv. At. Mol. Opt. Phys. 49, 373 (2003).

[4] M. Yu. Kuchiev, Pis'ma Zh. Eksp. Teor. Fiz. 45, 319 (1987) [JETP Lett. 45, 404 (1987)], http://www.jetpletters.ac.ru/ ps/1241/article_18763.pdf.

[5] K. J. Schafer, B. Yang, L. F. DiMauro, and K. C. Kulander, Phys. Rev. Lett. 70, 1599 (1993).

[6] P. B. Corkum, Phys. Rev. Lett. 71, 1994 (1993).

[7] D. B. Milosevic and F. Ehlotzky, Phys. Rev. A 65, 042504 (2002).

[8] A. N. Zheltukhin, N. L. Manakov, A. V. Flegel, and M. V. Frolov, Pis'ma Zh. Eksp. Teor. Fiz. 94, 641 (2011) [JETP Lett. 94, 599 (2011)].

[9] N. L. Manakov, A. F. Starace, A. V. Flegel, and M. V. Frolov, Pis'ma Zh. Eksp. Teor. Fiz. 76, 316 (2002) [JETP Lett. 76, 258 (2002)].

[10] A. Čerkić and D. B. Milošević, Phys. Rev. A 70, 053402 (2004).

[11] A. V. Flegel, M. V. Frolov, N. L. Manakov, and A. F. Starace, Phys. Lett. A 334, 197 (2005).

[12] A. Čerkić, M. Busuladžić, E. Hasović, A. GazibegovićBusuladžić, S. Odžak, K. Kalajdžić, and D. B. Milošević, Phys. Scr. T 149, 014044 (2012).

[13] A. V. Flegel, M. V. Frolov, N. L. Manakov, and A. N. Zheltukhin, J. Phys. B 42, 241002 (2009).

[14] F. V. Bunkin and M. V. Fedorov, Zh. Eksp. Teor. Fiz. 49, 1215 (1965) [Sov. Phys. JETP 22, 844 (1965)].

[15] N. M. Kroll and K. M. Watson, Phys. Rev. A 8, 804 (1973).

[16] A. D. Shiner, B. E. Schmidt, C. Trallero-Herrero, H. G. Wörner, S. Patchkovskii, P. B. Corkum, J.-C. Kieffer, F. Légaré, and D. M. Villeneuve, Nat. Phys. 7, 464 (2011).

[17] M. Okunishi, T. Morishita, G. Prumper, K. Shimada, C. D. Lin, S. Watanabe, and K. Ueda, Phys. Rev. Lett. 100, 143001 (2008).

[18] D. Ray et al., Phys. Rev. Lett. 100, 143002 (2008).

[19] T. Morishita, A. T. Le, Z. Chen, and C. D. Lin, Phys. Rev. Lett. 100, 013903 (2008).

[20] C. D. Lin, A. T. Le, Z. Chen, T. Morishita, and R. Lucchese, J. Phys. B 43, 122001 (2010).

[21] M. V. Frolov, N. L. Manakov, E. A. Pronin, and A. F. Starace, Phys. Rev. Lett. 91, 053003 (2003).

[22] M. V. Frolov, N. L. Manakov, and A. F. Starace, Phys. Rev. A 78, 063418 (2008).

[23] M. V. Frolov, N. L. Manakov, T. S. Sarantseva, and A. F. Starace, J. Phys. B 42, 035601 (2009).
[24] M. V. Frolov, N. L. Manakov, T. S. Sarantseva, M. Yu. Emelin, M. Yu. Ryabikin, and A. F. Starace, Phys. Rev. Lett. 102, 243901 (2009).

[25] M. V. Frolov, N. L. Manakov, and A. F. Starace, Phys. Rev. A 79, 033406 (2009).

[26] M. V. Frolov, N. L. Manakov, A. A. Silaev, N. V. Vvedenskii, and A. F. Starace, Phys. Rev. A 83, 021405(R) (2011).

[27] M. V. Frolov, D. V. Knyazeva, N. L. Manakov, A. M. Popov, O. V. Tikhonova, E. A. Volkova, Ming-Hui Xu, Liang-You Peng, Liang-Wen Pi, and A. F. Starace, Phys. Rev. Lett. 108, 213002 (2012).

[28] N. L. Manakov, A. F. Starace, A. V. Flegel, and M. V. Frolov, Pis'ma Zh. Eksp. Teor. Fiz. 87, 99 (2008) [JETP Lett. 87, 92 (2008)].

[29] S. P. Andreev, B. M. Karnakov, and V. D. Mur, Teor. Mat. Fiz. 64, 287 (1985) [Theor. Math. Phys. 64, 838 (1985)].

[30] L. D. Landau and E. M. Lifshitz, Quantum Mechanics (Nonrelativistic Theory), 4th ed. (Pergamon, Oxford, 1992).

[31] N. L. Manakov, V. D. Ovsiannikov, and L. P. Rapoport, Phys. Rep. 141, 319 (1986).

[32] N. L. Manakov and A. G. Fainshtein, Teor. Mat. Fiz. 48, 375 (1981) [Theor. Math. Phys. 48, 815 (1981)].

[33] D. B. Milošević, Phys. Rev. A 53, 619 (1996).

[34] L. B. Madsen and K. Taulbjerg, J. Phys. B 31, 4701 (1998).

[35] N. Bleistein and R. Handelsman, Asymptotic Expansions of Integrals (Dover, New York, 1986).

[36] R. Wong, Asymptotic Approximations of Integrals (Academic, Boston, 1989).

[37] M. Abramowitz and I. A. Stegun, eds., Handbook of Mathematical Functions (Dover, New York, 1965).

[38] A. V. Flegel, M. V. Frolov, N. L. Manakov, and A. F. Starace, J. Phys. B 38, L27 (2005).

[39] A. I. Nikishov and V. I. Ritus, Zh. Eksp. Teor. Fiz. 46, 776 (1964) [Sov. Phys. JETP 19, 529 (1964)].

[40] A. Čerkić and D. B. Milošević, Phys. Rev. A 73, 033413 (2006).

[41] Note that TDER theory, used in this paper, can be easily generalized to account for two (e.g., $s$ and $p$ ) phase shifts, as was done in the TDER theory for bound-state problems in Ref. [42].

[42] M. V. Frolov, N. L. Manakov, T. S. Sarantseva, and A. F. Starace, Phys. Rev. A 83, 043416 (2011).

[43] A. V. Flegel, M. V. Frolov, N. L. Manakov, and A. F. Starace, Phys. Rev. Lett. 102, 103201 (2009).

[44] D. A. Varshalovich, A. N. Moskalev, and V. K. Khersonskii, Quantum Theory of Angular Momentum (World Scientific, Singapore, 1988). 